\title{
Extended diffeomorphism algebras in (quantum) gravitational physics 1
}

\author{
V. Aldayd and J.L. Jaramilld
}

- Instituto de Astrofísica de Andalucía (CSIC), Apartado Postal 3004, 18080 Granada, Spain.

- Instituto Carlos I de Física Teórica y Computacional, Facultad de Ciencias, Universidad de Granada, Campus de Fuentenueva, Granada 18002, Spain.

\begin{abstract}
We construct an explicit representation of the algebra of local diffeomorphisms of a manifold with realistic dimensions. This is achieved in the setting of a general approach to the (quantum) dynamics of a physical system which is characterized by the fundamental role assigned to a basic underlying symmetry. The developed mathematical formalism makes contact with the relevant gravitational notions by means of the addition of some extra structure. The specific manners in which this is accomplished, together with their corresponding physical interpretation, lead to different gravitational models. Distinct strategies are in fact briefly outlined, showing the versatility of the present conceptual framework.
\end{abstract}

\section{Introduction}

A model for space-time dynamics in 1+1-dimensions was introduced in [1]. The present paper studies the necessary extension to realistic dimensions of the involved mathematical structures together with their physical implications.

The original work was conceived as a directly quantum formulation of the physical system, rather than a quantization of a previously devised classical theory. The rationale for such a construction was the fundamental role attached to the notion of symmetry in the characterization of the dynamics. Specifically, group cohomology governing the structure of the central extensions became the guiding principle in the identification of those dynamical degrees of freedom building the phase space. The choice of the abstract Virasoro algebra as the basic symmetry, led to a quantum theory making contact with two-dimensional Polyakov gravity [2] at the semi-classical level. The main achievements of that particular model were the recovery of a space-time notion out of the symmetry itself without its explicit introduction into scheme from the very beginning, on the one hand, and the gain of dynamical character by some diffeomorphisms thus entailing a quantum breakdown of their associated gauge invariance, on the other hand.

\footnotetext{
${ }^{1}$ Work partially supported by the DGICYT.

${ }^{2}$ E-mail: valdaya@iaa.es

${ }^{3}$ E-mail: jarama@iaa.es
} 
When extending this kind of analysis to systems with support on higher-dimensional spacetimes, we can identify two conceptually different levels of generalization. The first one, structural in nature, deals with the construction of the mathematical formalism supporting the new model. We shall address this problem by firstly identifying a Lie algebra which properly generalizes the Virasoro one, and then constructing the quantum and semi-classical dynamics that it provides. This task will be achieved by using the so-called Group Approach to Quantization (GAQ) [3], as was the case in [1]. The second level refers to the physical interpretation of that mathematical formalism. The intuition provided in the two-dimensional case by the Polyakov action will be definitively lost in the higher-dimensional model, and therefore new links with a metric notion will be needed. Our specific goal at this level will be the implementation of the quantum breakdown of diffeomorphism invariance at the quantum level, perhaps the most fundamental feature in this approach. With regard to the space-time recovery from the group structure we shall only dwell on the most simple situation in which no critical conditions in the cohomology parameters (i.e. anomalies) occur. Adopting this attitude, we are essentially leaving this specific issue open for future research.

Apart from the above-described line of reasoning, the present considerations make an interesting contact with the research program initiated in [⿴囗十]. A novel approach to the problem of mixing between space-time and internal gauge interactions was proposed with support on a revisited gauge principle which implies a non-trivial interplay between the corresponding local current algebras. The restriction in these works to a Particle-Mechanical setting freezes the dynamics corresponding to those local currents, therefore hiding their relevance. Nevertheless, these intrinsic dynamics prove to be an essential ingredient in the GAQ treatment of gauge interactions [5, 6] at the Field level (see below). In particular, gravitational interaction is addressed in [4] by making local the translation subgroup [7] which leads to a current algebra isomorphic to that of local diffeomorphisms (see subsection 2.2). Therefore the dynamics tied to this diffeomorphism algebra provides an intermediate step fundamental in the understanding of the proposed interaction mixing for field degrees of freedom, constituting a secondary but significant goal of the present paper.

The structure of the paper is as follows. Section 2 is divided in two subsections. First one briefly presents the fundamentals of the GAQ, the technique we use to construct the dynamical system out of a Lie symmetry. In the second one, the rigour of the presentation is significatly relaxed in order to motivate the relevant algebra we are using as a starting point. It also includes a brief explanation on the role of local gauge groups in the GAQ treatment of gauge theories. Section 3 recovers a more formal style and includes our main results, i.e., the dynamics associated with the Lie algebra proposed in the previous section. This will be done both at the semi-classical and quantum level. In particular, a maximum-weight representation of an (noncentrally) extended diffeomorphism algebra will be explicitly constructed. Section 4 contains the corresponding physical discussion and, finally, the conclusions will be presented in section 5 .

\section{General setting}

\subsection{Technical formalism: GAQ}

We briefly describe the basics of the Group Approach to Quantization (GAQ), a formalism that surpasses its role as a technichal device, deeply influencing and guiding the conceptual 
understanding of the physical dynamics construction.

The starting point is a Lie group $\tilde{G}$ with a $U(1)$-principal bundle structure with base $G$. This bundle possesses a connection 1-form $\Theta$ which generalizes the Poincaré-Cartan form $\Theta_{P C}$, i.e. the (pre-)contact form of time-dependent dynamics. This connection also plays a main role in the quantization procedure, and is (naturally) selected among the components of the left-invariant, Lie-algebra valued, Maurer-Cartan 1-form, in such a way that $\Theta(\Xi)=1$ and $\Theta\left(\tilde{X}_{i}^{L}\right)=0$, where $\Xi$ is the vertical (or fundamental) vector field of the principal bundle and $\tilde{X}_{i}^{L}$ are the rest of the independent left-invariant generators in a basis of the Lie algebra.

Taking $\tilde{G}$ as a central extension of $G$ by $U(1)$ (the tilde will denote extended objects throughout) we consider the space of complex functions $\Psi$ on $\tilde{G}$ satisfying the $U(1)$-equivariance condition, i.e. $\Psi(\zeta * g)=\zeta \Psi(g), \zeta \in U(1), g \in \tilde{G}$. On this space, the left translations on $\tilde{G}$ (generated by right-invariant vector fields $\tilde{X}^{R}$ ) realize a representation of the group which is in general reducible. Right translations, generated by left-invariant vector fields $\tilde{X}^{L}$, do commute with the left ones on any Lie group. Therefore, certain subgroups, called polarization subgroups $G_{\mathcal{P}}$, can be chosen to perform a reduction of the representation through the so-called polarization conditions, following the language of Geometric Quantization [9], which are implemented by left-invariant vector fields. This defines a reduced subspace of wave functions $\mathcal{H}$ satisfying $\Psi\left(g * G_{\mathcal{P}}\right)=\Psi(g)$ (or infinitesimally $L_{\tilde{X}^{L}} \Psi=0, \forall \tilde{X}^{L} \in \mathcal{G}_{\mathcal{P}}$, where $\mathcal{G}_{\mathcal{P}}$ is the Lie algebra of $G_{\mathcal{P}}$ ).

In the finite-dimensional case, the Hilbert space structure on the space of wave functions is provided by the invariant Haar-like measure constructed from the exterior product of the leftinvariant canonical form components, $\Omega^{L} \equiv \theta^{L g^{1}} \wedge \theta^{L g^{2}} \wedge \ldots$ On the reduced space $\mathcal{H}$ (at least for the case of first-order polarizations), the existence of a quasi-invariant measure is granted, since the wave functions have support on $G / G_{\mathcal{P}}$, which is a homogeneous space. However we shall deal here with an infinite-dimensional symmetry whose associated representation space is given by an orbit of the group through a (unique) vacuum state. This allows the introduction of a Hilbert product by fixing the normalization of that vacuum and imposing a rule of adjointness for the basic operators.

The classical theory for the system is easily recovered by defining the Noether invariants as $\mathcal{N}_{g^{i}} \equiv i_{\tilde{X}_{g^{i}}} \Theta$. They are in fact invariant under the evolution generated by vector fields inside $\mathcal{G}_{\Theta} \equiv \operatorname{Ker} \Theta \cap \operatorname{Kerd} \Theta$, which define the generalized equations of motion. The classical phase space is given by $G / G_{\Theta}$ (see [3] and references therein). A Poisson bracket can be introduced (defined by $d \Theta$ ) in such a way that the Noether invariants generate a Lie algebra isomorphic to that of $\tilde{G}$.

\subsection{Starting algebra}

The choice of the starting Lie algebra, defining the fundamental symmetry underlying the system, will be guided by the attempt to implement our basic goals.

\section{Generalization of Virasoro algebra}

Firstly, the generalization of the Virasoro algebra, together with the desire to analyse a potential physical breakdown of the diffeomorphism invariance in realistic space-time dimensions, suggests the adoption of the local diffeomorphism algebra of a $d$-dimensional manifold $M(d \geq 2)$ as a natural departure point. This algebra, denoted by $V$ ect $(M)$ in [10], is simply given by vector 
fields on $M$ with the natural Lie product,

$$
\left[\mathcal{L}_{\eta}, \mathcal{L}_{\xi}\right]=\mathcal{L}_{[\eta, \xi]}
$$

where $[\eta, \xi]=\left(\eta^{\mu} \partial_{\mu} \xi^{\nu}-\xi^{\mu} \partial_{\mu} \eta^{\nu}\right) \partial_{\nu}$. The analysis of the associated dynamics is more easily developed by fixing a basis for the vector fields. When choosing $L_{\mu}(\mathbf{m})=i e^{-i m_{\rho} x^{\rho}} \partial_{\mu}$, with $\mathbf{m}$ a $d$-dimensional vector of integer entries, (1) is written,

$$
\left[L_{\mu}(\mathbf{m}), L_{\nu}(\mathbf{n})\right]=n_{\mu} L_{\nu}(\mathbf{m}+\mathbf{n})-m_{\nu} L_{\mu}(\mathbf{m}+\mathbf{n}) .
$$

The cohomological analysis of this algebra rules out the possibility of non-trivial central extensions $(d \geq 2)$, which is not a fundamental drawback for the construction of the corresponding dynamics, as will be explicitly shown at the end of this section. Non-central extensions must be considered if one wants to enrich the dynamics, entailing the introduction of new fields in the model. A particular class of these extensions, the tensorial ones, are studied and classified in [12], 13] providing a variety of possibilities from which to choose. Here we shall consider the simplest extension, represented by a generator $S^{\rho}(\mathbf{m})$. This option constitutes in fact a direct generalization of the cubic cocycle of the Virasoro algebra, reducing to it in the one-dimensional case (to be precise, a closure condition must be imposed to $S^{\rho}(\mathbf{m})$ ). The Lie algebra is given by:

$$
\begin{aligned}
& {\left[L_{\mu}(\mathbf{m}), L_{\nu}(\mathbf{n})\right]=n_{\mu} L_{\nu}(\mathbf{m}+\mathbf{n})-m_{\nu} L_{\mu}(\mathbf{m}+\mathbf{n})-m_{\mu} n_{\nu}\left(m_{\rho}-n_{\rho}\right) S^{\rho}(\mathbf{m}+\mathbf{n})} \\
& {\left[L_{\mu}(\mathbf{m}), S^{\nu}(\mathbf{n})\right]=n_{\mu} S^{\nu}(\mathbf{m}+\mathbf{n})+\delta_{\mu}^{\nu} m_{\rho} S^{\rho}(\mathbf{m}+\mathbf{n})}
\end{aligned}
$$

\section{Contact with the interaction mixing problem}

Secondly, and in order to address our purpose of relating this algebra with the works in «4, let us briefly sketch a comparison between the ways in which field degrees of freedom are handled in the standard approach to (Yang-Mills) gauge theories and in GAQ.

In an oversimplified version of the former, which omits all technical and conceptual subtleties, the field degrees of freedom are constructed in terms of the components $A_{\mu}^{a}$ (gauge fields) of a connection on a principal bundle, with $U$ as the structure group and the space-time $M$ as its base. These modes couple to the matter degrees of freedom, living on an associated vector bundle, by means of a the so-called minimal coupling, technically implemented by replacing the partial derivatives in the matter Lagrangian with a covariant derivative defined in terms of the connection (very roughly, $\partial_{\mu} \rightarrow\left(\partial_{\mu}+A_{\mu}\right)$ ). The Lagrangian giving the intrinsic dynamics of the gauge fields is defined as a function of the curvature $F_{\mu \nu}$ of the connection form [14]. This description of the system is overcomplete since not all the field degrees $A_{\mu}^{a}$ are in fact physical. Two connections $A_{\mu}^{\prime}$ and $A_{\mu}$ are physically equivalent if they are related by the action of the gauge group (locally identifiable with $\operatorname{Map}(M, U)$ ), $A_{\mu}^{\prime}=u^{-1} A_{\mu} u+u^{-1} d u$. Taking quotient by the orbits of the gauge group, we obtained the reduced or physical phase space.

The whole spirit and structural description changes in the GAQ. Instead of a space of field degrees of freedom parametrized by $A_{\mu}$, on which a Lagrangian $L$ is defined and which must be reduced according to the action of a given gauge group, in GAQ all these elements must be merged into a unique centrally-extended group $\tilde{G}$. The dynamics, as well as the identification of the physical degrees of freedom, must follow from the algorithm described in the previous subsection. There is no obvious way of endowing the connection components $A_{\mu}^{a}$ with a group 
law structure, closing, in addition, a Lie symmetry with the gauge group $M a p(M, U)$. An explicit solution to this problem, not necessarily unique, is presented in [5, 6]. The price we must pay for closing such a unified Lie structure is the loss of a clean distinction at the group law level between the degrees of freedom corresponding to the gauge fields (the "connections") and the parameters corresponding to the gauge group. These modes are intertwined in a subtle manner in such a way that a transfer of dynamical content occurs between them. In order to fix ideas we comment on the simplest case of a local $U(1)$ gauge group in the unified treatment of the electromagnetic and Proca fields [5]. Working in momentum space and parametrizing the gauge fields by $a_{\mu}(k)$ and $a_{\mu}^{\dagger}(k)$, and the $U(1)$ parameters by $\phi(k)$ and $\phi^{+}(k)$, the corresponding group law implies at the Lie algebra level the following commutators,

$$
\begin{aligned}
{\left[\tilde{X}_{a_{\mu}^{\dagger}(k)}^{L}, \tilde{X}_{a_{\mu}\left(k^{\prime}\right)}^{L}\right] } & =i \eta^{\mu \nu} \Delta_{k k^{\prime}} \Xi \\
{\left[\tilde{X}_{\phi^{\dagger}(k)}^{L}, \tilde{X}_{\phi\left(k^{\prime}\right)}^{L}\right] } & =i k^{2} \Delta_{k k^{\prime}} \Xi \\
{\left[\tilde{X}_{a_{\mu}(k)}^{L}, \tilde{X}_{\phi^{\dagger}\left(k^{\prime}\right)}^{L}\right] } & =k^{\mu} \Delta_{k k^{\prime}} \Xi \\
{\left[\tilde{X}_{a_{\mu}^{\dagger}(k)}^{L}, \tilde{X}_{\phi\left(k^{\prime}\right)}^{L}\right] } & =k^{\mu} \Delta_{k k^{\prime}} \Xi,
\end{aligned}
$$

where $\Delta_{k k^{\prime}}=2 k^{0} \delta^{3}\left(k-k^{\prime}\right)$ is the generalized delta function on the positive sheet of the mass hyperboloid. The second commutator explicitly shows the non-empty symplectic content of $\phi(k)$, whereas the third and forth implies the above-mentioned mixing between the $a_{\mu}(k)$ and $\phi(k)$ variables. The diagonalization of the cocycle when determining the canonical symplectic variables entails the transfer of dynamical content between modes. The generators playing the role of authentic gauge tranformations in this setting (they leave the reduced physical phase space invariant pointwise) are in fact,

$$
\begin{aligned}
\tilde{X}_{c(k)}^{L} & =\tilde{X}_{\phi(k)}^{L}+i k_{\mu} \tilde{X}_{a_{\mu}(k)}^{L} \\
\tilde{X}_{c^{\dagger}(k)}^{L} & =\tilde{X}_{\phi^{\dagger}(k)}^{L}+i k_{\mu} \tilde{X}_{a_{\mu}^{\dagger}(k)}^{L}
\end{aligned}
$$

which imply the equivalence condition,

$$
a_{\mu}(k) \sim a_{\mu}(k)+i k_{\mu} c(k), \phi(k) \sim \phi(k)+c(k)
$$

together with its hermitian conjugate, where $c(k)$ is the evolution parameter of the $\tilde{X}_{c(k)}^{L}$ vector field. They simply represent the momentum version of the standard gauge transformations.

The only aim in the precedent discussion was that of underlying the importance of a good understanding of the (abstract) intrinsic dynamics associated with the local gauge group, determined by its extensions, when one is addressing gauge theories in the GAQ formalism.

Coming back to the idea of mixing external and internal interactions as described in [4], the relevant gauge invariance groups were determined from the local current algebras $\mathcal{F}(M) \otimes \mathcal{G}$, where $\mathcal{F}(M)$ is the real functions on $M$ and $\mathcal{G}$ the Lie algebra of the rigid part of the gauge group. Choosing a realization $\left\{X_{a}\right\}$ for the latter, we have:

$$
\left[f \otimes X_{a}, g \otimes X_{b}\right]=\left(f L_{X_{a}} g\right) \otimes X_{b}-\left(g L_{X_{b}} f\right) \otimes X_{a}+(f g) \otimes\left[X_{a}, X_{b}\right] .
$$

The gravitational interaction was addressed by making local the rigid translation group. Choosing $\partial_{\mu}$ as a realization for its generators, the corresponding current algebra turns out to be 
isomorphic to the local diffeomorphism algebra (11). Therefore, in the study of Gravity as a gauge interaction with a gauge group obtained by making local the space-time translations ([0] and first part of IIIA in [8]), the analysis of the intrinsic dynamics derived from the diffeomorphism algebra plays a fundamental role in a GAQ setting. The modes $L_{\mu}(\mathbf{m})$ are just the analogue to $\phi(k)$ in the electromagnetic case. Of course, it represents only an intermediate step, since the gauge fields analogue to $a_{\mu}(k)$ fl should be consistenly introduced at a second stage.

The mixing of gravitational and electromagnetic interactions in 酒 occurred through the weaving together the corresponding current algebras. In order to contemplate such a possibility, we introduce the new generators $f \otimes \Xi$ corresponding to the local $U(1)$. In the momentum space we are dealing with, their expression is $A(\mathbf{m})$ and the new commutators are given by

$$
\begin{aligned}
{\left[L_{\mu}(\mathbf{m}), A(\mathbf{n})\right] } & =n_{\mu} A(\mathbf{m}+\mathbf{n}) \\
{[A(\mathbf{m}), A(\mathbf{n})] } & =\left(n_{\rho}-m_{\rho}\right) S^{\rho}(\mathbf{m}+\mathbf{n}),
\end{aligned}
$$

where we have taken advantage of the possibility of introducing a new (and independent) extension in the $U(1)$ sector implemented by the already present $S^{\rho}(\mathbf{m})$ generators. Therefore, our starting algebra is given by commutators (3) + (8) .

\section{Central extensions}

Since we need a $U(1)$-centrally extended group in order to employ the GAQ, the starting Lie algebra should present central terms in the commutators. The central terms that cannot be eliminated by a linear change of basis in the Lie algebra are controlled at the group level foy the non-trivial two-cocycles in the second cohomology group of the non-extended Lie group $G$, $H^{2}(G, U(1))$. But these are not the only cocycles capable of creating dynamics. In fact, and it is specially important in the case of Lie groups with trivial cohomology, those coboundaries $\xi_{\lambda}$ generated by a function on the group $\lambda$ such that $\left.\frac{\partial \lambda(g)}{\partial g^{i}}\right|_{g=e} \neq 0$ lead to dynamics on phases spaces which are symplectomorphic to the coadjoint orbits of the group. Of course, these coboundaries generate central terms at the algebra level that could be eliminated by linear changes, so they are consistently trivial from the Lie structure point of view. Nevertheless, they have crucial consequences on the polarization conditions and therefore, at the representation level. We can reverse the reasoning and consider that, once the algebra has been (trivially or not) extended by a central $U(1)$ group, a linear change in the algebra involving the central generator will be associated with one of those coboundaries creating dynamics. These subclasses inside $H^{2}(G, U(1))$ were first introduced in [17] associated with some coboundaries $\xi_{\lambda}$, which become non-trivial cocycles $\xi_{c}$ after an Inönü-Wigner contraction takes place, and fully studied in connection with coadjoint orbits in [11]. The simplest example of the latter is the Poincaré group whose pseudo-cohomology goes to the true cohomology of the Galilei group [18]. For finitedimensional semi-simple groups, pseudo-cohomology is also related to the Cech cohomology of the generalized Hopf fibrations by the Cartan subgroups $H, G \rightarrow G / H$ [19].

The algebra (3) $+(8)$ we are using as the starting point does not present the central extension structure we need in order to derive a dynamics. In fact, no non-trivial extensions exists when the space-time dimension is over one. Therefore, we take advantage of the above-discussed

\footnotetext{
${ }^{4}$ In principle several possibilities arise ranging from metric variables to connection ones in the spirit of Ashtekar variables.

${ }^{5}$ In this general remark we shall not address the differences between Lie algebra and Lie group cohomology.
} 
pseudo-cohomology and we add a new central generator $\Xi$ to the Lie algebra in a direct-sum way. We then perform the linear change

$$
\begin{aligned}
A(\mathbf{m}) & \mapsto A(\mathbf{m})+\alpha \delta(\mathbf{m}) \Xi \\
S^{\rho}(\mathbf{m}) & \mapsto S^{\rho}(\mathbf{m})+C^{\rho} \delta(\mathbf{m}) \Xi \\
L_{\mu}(\mathbf{m}) & \mapsto L_{\mu}(\mathbf{m})+\tilde{C}_{\mu} \delta(\mathbf{m}) \Xi
\end{aligned}
$$

which will give rise to one of the dynamically non-trivial coboundaries, after exponentiation.

\section{Dynamics}

As we have seen, the starting point for the whole construction is the explicit group law of the Lie symmetry defining the physical system. In the previous section we only worked at the algebra level. Then, the first step now is to exponentiate it to a finite group law. This entails the complications derived from dealing with infinite-dimensional Lie algebras. We shall face this difficulty by using a perturbative order-by-order construction of a formal group law [15], as described in 16.

Using an index $\mu(\mathbf{m})$ for $L_{\mu}(\mathbf{m}), \tilde{\mu}(\mathbf{m})$ for $S^{\mu}(\mathbf{m}), \mathbf{m}$ for $A(\mathbf{m})$ and $\varphi$ for $\Xi$, the structure constants in this basis are (there is a harmless global redefinition by a minus sign related to the particular exponentiation technique we are going to employ):

$$
\begin{aligned}
C_{\mu(\mathbf{m}) \nu(\mathbf{n})}^{\rho(\mathbf{r})} & =-\left(\mathbf{n}_{\mu} \delta_{\nu}^{\rho}-\mathbf{m}_{\nu} \delta_{\mu}^{\rho}\right) \delta(\mathbf{m}+\mathbf{n}-\mathbf{r}) \\
C_{\mu(\mathbf{m}) \nu(\mathbf{n})}^{\tilde{\rho}(\mathbf{r})} & =m_{\mu} n_{\nu}\left(m_{\rho}-n_{\rho}\right) \delta(\mathbf{m}+\mathbf{n}-\mathbf{r}) \\
C_{\mu(\mathbf{m}) \nu(\mathbf{n})}^{\varphi} & =-\left[-m_{\mu} n_{\nu}\left(m_{\rho}-n_{\rho}\right) C^{\rho}+\left(n_{\mu} \delta_{\nu}^{\rho}-m_{\nu} \delta_{\mu}^{\rho}\right) \tilde{C}_{\rho}\right] \delta(\mathbf{m}+\mathbf{n}) \\
C_{\mu(\mathbf{m}) \tilde{\nu}(\mathbf{n})}^{\tilde{\rho}(\mathbf{r})} & =-\left(n_{\mu} \delta_{\rho}^{\nu}-m_{\nu} \delta_{\rho}^{\mu}\right) \delta(\mathbf{m}+\mathbf{n}-\mathbf{r}) \\
C_{\mu(\mathbf{m}) \tilde{\nu}(\mathbf{n})}^{\varphi} & =-\left(n_{\mu} \delta_{\rho}^{\nu}-m_{\nu} \delta_{\rho}^{\mu}\right) C^{\rho} \delta(\mathbf{m}+\mathbf{n}) \\
C_{\mathbf{m n}}^{\tilde{\rho}(\mathbf{r})} & =-\left(n_{\rho}-m_{\rho}\right) \delta(\mathbf{m}+\mathbf{n}-\mathbf{r}) \\
C_{\mathbf{m n}}^{\varphi} & =-\left(n_{\rho}-m_{\rho}\right) c_{2} C^{\rho} \delta(\mathbf{m}+\mathbf{n}) \\
C_{\mu(\mathbf{m}) \mathbf{n}}^{\mathbf{r}} & =-n_{\mu} \delta(\mathbf{m}+\mathbf{n}-\mathbf{r}) \\
C_{\mu(\mathbf{m}) \mathbf{n}}^{\varphi} & =-\alpha n_{\mu} \delta(\mathbf{m}+\mathbf{n})
\end{aligned}
$$

We use them to construct a formal group law, $g^{\prime \prime}=g^{\prime} * g$, up to the third-order in the group variables. We associate the variable $l^{\mu}(\mathbf{m})$ with the generator $L_{\mu}(\mathbf{m}), s^{\mu}(\mathbf{m})$ with $S_{\mu}(\mathbf{m}), a(\mathbf{m})$ with $A(\mathbf{m})$ and $\varphi$ with $\Xi$, obtaining:

$$
\begin{aligned}
l^{\prime \prime \rho}(\mathbf{r}) & =l^{\prime \rho}(\mathbf{r})+l^{\rho}(\mathbf{r})+\frac{1}{2} C_{\mu(\mathbf{m}) \nu(\mathbf{n})}^{\rho(\mathbf{r})} l^{\prime \mu}(\mathbf{m}) l^{\nu}(\mathbf{n}) \\
& +\frac{1}{8} C_{\nu(\mathbf{n}) \mu(\mathbf{m})}^{\rho(\mathbf{r})} C_{\gamma(\mathbf{q}) \sigma(\mathbf{s})}^{\mu(\mathbf{m})} l^{\prime \nu}(\mathbf{n}) l^{\prime \gamma}(\mathbf{q}) l^{\sigma}(\mathbf{s})+\ldots \\
s^{\prime \prime \rho}(\mathbf{r}) & =s^{\prime \rho}(\mathbf{r})+s^{\rho}(\mathbf{r})+\frac{1}{2} C_{\mu(\mathbf{m}) \nu(\mathbf{n})}^{\tilde{\rho}(\mathbf{r})} l^{\prime \mu}(\mathbf{m}) l^{\nu}(\mathbf{n}) \\
& +C_{\mu(\mathbf{m}) \tilde{\nu}(\mathbf{n})}^{\tilde{\rho}(\mathbf{r})} l^{\prime \mu}(\mathbf{m}) s^{\nu}(\mathbf{n})+\frac{1}{2} C_{\mathbf{m} \mathbf{n}}^{\tilde{\rho}(\mathbf{r})} a^{\prime}(\mathbf{m}) a(\mathbf{n})
\end{aligned}
$$




$$
\begin{aligned}
+ & \frac{1}{2}\left[\left(\frac{1}{4} C_{\nu(\mathbf{n}) \mu(\mathbf{m})}^{\tilde{\rho}(\mathbf{r})} C_{\gamma(\mathbf{q}) \sigma(\mathbf{s})}^{\mu(\mathbf{m})}+\frac{1}{2} C_{\nu(\mathbf{n}) \tilde{\mu}(\mathbf{m})}^{\tilde{\rho}(\mathbf{r})} C_{\gamma(\mathbf{q}) \sigma(\mathbf{s})}^{\tilde{\mu}(\mathbf{m})}\right) l^{\prime \nu}(\mathbf{n}) l^{\prime \gamma}(\mathbf{q}) l^{\sigma}(\mathbf{s})\right. \\
& \left.+C_{\nu(\mathbf{n}) \tilde{\mu}(\mathbf{m})}^{\tilde{\rho}(\mathbf{r})} C_{\gamma(\mathbf{q}) \tilde{\sigma}(\mathbf{s})}^{\tilde{\mu}(\mathbf{m})} l^{\prime \nu}(\mathbf{n}) l^{\prime \gamma}(\mathbf{q}) s^{\sigma}(\mathbf{s})+\frac{1}{2} C_{\mathbf{n} \mathbf{m}}^{\tilde{\rho}(\mathbf{r})} C_{\gamma(\mathbf{q}) \mathbf{s}}^{\mathbf{m}} l^{\prime \gamma}(\mathbf{q}) a^{\prime}(\mathbf{n}) a(\mathbf{s})\right]+\ldots \\
a^{\prime \prime}(\mathbf{p}) & =a^{\prime}(\mathbf{p})+a(\mathbf{p})+C_{\mu(\mathbf{m}) \mathbf{n}}^{\mathbf{p}} l^{\prime \mu}(\mathbf{m}) a(\mathbf{n})+\frac{1}{2} C_{\nu(\mathbf{n}) \mathbf{m}}^{\mathbf{p}} C_{\gamma(\mathbf{q}) \mathbf{s}}^{\mathbf{m}} l^{\prime \nu}(\mathbf{n}) l^{\prime \gamma}(\mathbf{q}) a(\mathbf{s})+\ldots \\
\varphi^{\prime \prime} & =\varphi^{\prime}+\varphi+\frac{1}{2} C_{\mu(\mathbf{m}) \nu(\mathbf{n})}^{\varphi} l^{\prime \mu}(\mathbf{m}) l^{\nu}(\mathbf{n})+C_{\mu(\mathbf{m}) \tilde{\nu}(\mathbf{m})}^{\varphi} l^{\prime \mu}(\mathbf{m}) s^{\nu}(\mathbf{n})+C_{\mu(\mathbf{m}) \mathbf{n}}^{\varphi} l^{\prime \mu}(\mathbf{m}) a(\mathbf{n}) \\
& +\frac{1}{2} C_{\mathbf{m} \mathbf{n}}^{\varphi} a^{\prime}(\mathbf{m}) a(\mathbf{n})+\frac{1}{2}\left[\left(\frac{1}{4} C_{\rho(\mathbf{r}) \mu(\mathbf{m})}^{\varphi} C_{\nu(\mathbf{n}) \sigma(\mathbf{s})}^{\mu(\mathbf{m})}+\frac{1}{2} C_{\rho(\mathbf{r}) \tilde{\mu}(\mathbf{m})}^{\varphi} C_{\nu(\mathbf{n}) \sigma(\mathbf{s})}^{\tilde{\mu}(\mathbf{m})}\right) l^{\prime \rho}(\mathbf{r}) l^{\prime \nu}(\mathbf{n}) l^{\sigma}(\mathbf{s})\right. \\
& +C_{\rho(\mathbf{r}) \tilde{\mu}(\mathbf{m})}^{\varphi} C_{\nu(\mathbf{n}) \tilde{\sigma}(\mathbf{s})}^{\tilde{\mu}(\mathbf{m})} l^{\prime \rho}(\mathbf{r}) l^{\prime \nu}(\mathbf{n}) s^{\sigma}(\mathbf{s})+C_{\rho(\mathbf{r}) \mathbf{m}}^{\varphi} C_{\nu(\mathbf{n}) \mathbf{s}}^{\mathbf{m}} l^{\prime \rho}(\mathbf{r}) l^{\prime \nu}(\mathbf{n}) a(\mathbf{s}) \\
& \left.+\frac{1}{2} C_{\mathbf{r m}}^{\varphi} C_{\nu(\mathbf{n}) \mathbf{s}}^{\mathbf{m}}\right] l^{\prime \nu}(\mathbf{n}) a^{\prime}(\mathbf{r}) a(\mathbf{s})+\ldots .
\end{aligned}
$$

Using this, we compute the left-invariant vector fields, $\tilde{X}_{(k)}^{L}(g)=\left.\frac{\partial g^{\prime \prime l}\left(g^{\prime}, g\right)}{\partial g^{(k)}}\right|_{g=e, g^{\prime}=g} \frac{\partial}{\partial g^{l}}$, yielding,

$$
\begin{aligned}
& \tilde{X}_{l^{\tau}(\mathbf{t})}^{L}=\frac{\partial}{\partial l^{\tau}(\mathbf{t})}+\left\{\frac{1}{2} C_{\mu(\mathbf{m}) \tau(\mathbf{t})}^{\rho(\mathbf{r})} l^{\mu}(\mathbf{m})+\frac{1}{8} C_{\nu(\mathbf{n}) \mu(\mathbf{m})}^{\rho(\mathbf{r})} C_{\gamma(\mathbf{q}) \tau(\mathbf{t})}^{\mu(\mathbf{m})} l^{\nu}(\mathbf{n}) l^{\gamma}(\mathbf{q})+\ldots\right\} \frac{\partial}{\partial l^{\rho}(\mathbf{r})} \\
& +\left\{\frac{1}{2} C_{\mu(\mathbf{m}) \tau(\mathbf{t})}^{\tilde{\rho}(\mathbf{r})} l^{\mu}(\mathbf{m})+\left(\frac{1}{8} C_{\nu(\mathbf{n}) \mu(\mathbf{m})}^{\tilde{\rho}(\mathbf{r})} C_{\gamma(\mathbf{q}) \tau(\mathbf{t})}^{\mu(\mathbf{m})}\right.\right. \\
& \left.\left.+\quad \frac{1}{4} C_{\nu(\mathbf{n}) \tilde{\mu}(\mathbf{m})}^{\tilde{\rho}(\mathbf{r})} C_{\gamma(\mathbf{q}) \tau(\mathbf{t})}^{\tilde{\mu}(\mathbf{m})}\right) l^{\nu}(\mathbf{n}) l^{\gamma}(\mathbf{q})+\ldots\right\} \frac{\partial}{\partial s^{\rho}(\mathbf{r})}+\left\{\frac{1}{2} C_{\mu(\mathbf{m}) \tau(\mathbf{t})}^{\varphi} l^{\mu}(\mathbf{m})\right. \\
& \left.+\left(\frac{1}{8} C_{\nu(\mathbf{n}) \mu(\mathbf{m})}^{\varphi} C_{\gamma(\mathbf{q}) \tau(\mathbf{t})}^{\mu(\mathbf{m})}+\frac{1}{4} C_{\nu(\mathbf{n}) \tilde{\mu}(\mathbf{m})}^{\varphi} C_{\gamma(\mathbf{q}) \tau(\mathbf{t})}^{\tilde{\mu}(\mathbf{m})}\right) l^{\nu}(n) l^{\gamma}(\mathbf{q})+\ldots\right\} \frac{\partial}{\partial \varphi} \\
& \tilde{X}_{s^{\tau}(\mathbf{t})}^{L}=\frac{\partial}{\partial l^{\tau}(\mathbf{t})}+\left\{C_{\mu(\mathbf{m}) \tilde{\tau}(\mathbf{t})}^{\tilde{\rho}(\mathbf{r})} l^{\mu}(m)+\frac{1}{2} C_{\nu(\mathbf{n}) \tilde{\mu}(\mathbf{m})}^{\tilde{\rho}(\mathbf{( r )}} C_{\gamma(\mathbf{q}) \tilde{\tau}(\mathbf{t})}^{\tilde{\mu}(\mathbf{m})} l^{\nu}(\mathbf{n}) l^{\gamma}(\mathbf{q})+\ldots\right\} \frac{\partial}{\partial s^{\rho}(\mathbf{r})} \\
& +\left\{C_{\mu(\mathbf{m}) \tilde{\tau}(\mathbf{t})}^{\varphi} l^{\mu}(\mathbf{m})+\frac{1}{2} C_{\nu(\mathbf{n}) \tilde{\mu}(\mathbf{m})}^{\varphi} C_{\gamma(\mathbf{q}) \tilde{\tau}(\mathbf{t})}^{\tilde{\mu}(\mathbf{m})} l^{\nu}(\mathbf{n}) l^{\gamma}(\mathbf{q})+\ldots\right\} \frac{\partial}{\partial \varphi} \\
& \tilde{X}_{a(\mathbf{t})}^{L}=\frac{\partial}{\partial a(\mathbf{t})}+\left\{C_{\mu(\mathbf{m}) \mathbf{t}}^{\mathbf{r}} l^{\mu}(\mathbf{m})+\frac{1}{2} C_{\nu(\mathbf{n}) \mathbf{m}}^{\mathbf{r}} C_{\gamma(\mathbf{q}) \mathbf{t}}^{\mathbf{m}} l^{\nu}(\mathbf{n}) l^{\gamma}(\mathbf{q})+\ldots\right\} \frac{\partial}{\partial a(\mathbf{r})} \\
& +\left\{\frac{1}{2} C_{\mathbf{m t}}^{\tilde{\rho}(\mathbf{r})} a(\mathbf{m})+\frac{1}{4} C_{\mathbf{q} \mathbf{m}}^{\tilde{\rho}(\mathbf{r})} C_{\nu(\mathbf{n}) \mathbf{t}}^{\mathbf{m}} l^{\nu}(\mathbf{n}) a(\mathbf{q})+\ldots\right\} \frac{\partial}{\partial s^{\rho}(\mathbf{p})} \\
& +\left\{\frac{1}{2} C_{\mathbf{m t}}^{\varphi} a(\mathbf{m})+C_{\mu(\mathbf{m}) \mathbf{t}}^{\varphi} l^{\mu}(\mathbf{m})+\frac{1}{2} C_{\rho(\mathbf{r}) \mathbf{m}}^{\varphi} C_{\nu(\mathbf{n}) \mathbf{t}}^{\mathbf{m}} l^{\rho}(\mathbf{r}) l^{\nu}(\mathbf{n})\right. \\
& \left.+\frac{1}{4} C_{\mathbf{n} \mathbf{m}}^{\varphi} C_{\rho(\mathbf{r}) \mathbf{t}}^{\mathbf{m}} \boldsymbol{l}^{\rho}(\mathbf{r}) a(\mathbf{n})+\ldots\right\} \frac{\partial}{\partial \varphi} \\
& \tilde{X}_{\varphi}^{L}=\frac{\partial}{\partial \varphi}
\end{aligned}
$$

and also the right-invariant vector fields $\tilde{X}_{(k)}^{R}(g)=\left.\frac{\partial g^{\prime \prime l}\left(g^{\prime}, g\right)}{\partial g^{\prime(k)}}\right|_{g^{\prime}=e, g=g} \frac{\partial}{\partial g^{l}}$,

$$
\tilde{X}_{l^{\tau}(\mathbf{t})}^{R}=\frac{\partial}{\partial l^{\tau}(\mathbf{t})}+\frac{1}{2} C_{\tau(\mathbf{t}) \nu(\mathbf{n})}^{\rho(\mathbf{r})} l^{\nu}(\mathbf{n}) \frac{\partial}{\partial l^{\rho}(\mathbf{r})}+\left(\frac{1}{2} C_{\tau(\mathbf{t}) \nu(\mathbf{n})}^{\tilde{\rho}(\mathbf{r})} l^{\nu}(\mathbf{n})+C_{\tau(\mathbf{t}) \tilde{\nu}(\mathbf{n})}^{\tilde{\rho}(\mathbf{r})} s^{\nu}(\mathbf{n})\right) \frac{\partial}{\partial s^{\rho}(\mathbf{r})}
$$




$$
\begin{aligned}
& +C_{\tau(\mathbf{t}) \mathbf{n}}^{\mathbf{r}} a(\mathbf{n}) \frac{\partial}{\partial a(\mathbf{r})}+\left(\frac{1}{2} C_{\tau(\mathbf{t}) \nu(\mathbf{n})}^{\varphi} l^{\nu}(\mathbf{n})+C_{\tau(\mathbf{t}) \tilde{\nu}(\mathbf{n})}^{\varphi} s^{\nu}(\mathbf{n})+C_{\tau(\mathbf{t}) \mathbf{n}}^{\varphi} a(\mathbf{n})\right) \frac{\partial}{\partial \varphi} \\
\tilde{X}_{s^{\tau}(\mathbf{t})}^{R} & =\frac{\partial}{\partial s^{\tau}(\mathbf{t})} \\
\tilde{X}_{a(\mathbf{t})}^{R} & =\frac{\partial}{\partial a(\mathbf{t})}+\frac{1}{2} C_{\mathbf{t n}}^{\tilde{\rho}(\mathbf{r})} a(\mathbf{n}) \frac{\partial}{\partial s^{\rho}(\mathbf{r})}+\frac{1}{2} C_{\mathbf{t n}}^{\varphi} a(\mathbf{n}) \frac{\partial}{\partial \varphi} \\
\tilde{X}_{\varphi}^{R} & =\frac{\partial}{\partial \varphi} .
\end{aligned}
$$

Analogously, the quantization one-form, $\Theta=\theta^{L^{(\varphi)}}=\left.\frac{\partial \varphi^{\prime \prime l}\left(g^{\prime}, g\right)}{\partial g^{(k)}}\right|_{g=g, g^{\prime}=g^{-1}} d g^{k}$ has the expression

$$
\begin{aligned}
\Theta & =\theta^{L(\varphi)}=d \varphi \\
& +\left\{-\frac{1}{2} C_{\mu(\mathbf{m}) \nu(\mathbf{n})}^{\varphi} l^{\mu}(\mathbf{m})+\left(\frac{1}{8} C_{\rho(\mathbf{r}) \mu(\mathbf{m})}^{\varphi} C_{\gamma(\mathbf{q}) \nu(\mathbf{n})}^{\mu(\mathbf{m})}+\frac{1}{4} C_{\rho(\mathbf{r}) \tilde{\mu}(\mathbf{m})}^{\varphi} C_{\gamma(\mathbf{q}) \nu(\mathbf{n})}^{\tilde{\mu}(\mathbf{m})}\right) l^{\rho}(\mathbf{r}) l^{\gamma}(\mathbf{q})\right\} d l^{\nu}(\mathbf{n}) \\
& +\left\{-C_{\mu(\mathbf{m}) \tilde{\nu}(\mathbf{n})}^{\varphi} l^{\mu}(\mathbf{m})+\frac{1}{2} C_{\rho(\mathbf{r}) \tilde{\mu}(\mathbf{m})}^{\varphi} C_{\gamma(\mathbf{q}) \tilde{\nu}(\mathbf{n})}^{\tilde{\mu}(\mathbf{m})} l^{\rho}(\mathbf{r}) l^{\gamma}(\mathbf{q})\right\} d s^{\nu}(\mathbf{n}) \\
& +\left\{-\frac{1}{2} C_{\mathbf{m} \mathbf{n}}^{\varphi} a(\mathbf{m})-C_{\mu(\mathbf{m}) \mathbf{n}}^{\varphi} l^{\mu}(\mathbf{m})+\frac{1}{4}\left(2 C_{\mathbf{m} \mathbf{n}}^{\varphi} C_{\rho(\mathbf{r}) \mathbf{q}}^{\mathbf{m}}+C_{\mathbf{q} \mathbf{m}}^{\varphi} C_{\rho(\mathbf{r}) \mathbf{n}}^{\mathbf{m}}\right) l^{\rho}(\mathbf{r}) a(\mathbf{q})\right. \\
& \left.+\frac{1}{2} C_{\rho(\mathbf{r}) \mathbf{m}}^{\varphi} C_{\gamma(\mathbf{q}) \mathbf{n}}^{\mathbf{m}} l^{\rho}(\mathbf{r}) l^{\gamma}(\mathbf{q})\right\} d a(\mathbf{n}) .
\end{aligned}
$$

\subsection{Semi-classical formalism}

Except for critical combinations of the pseudo-cohomology parameters (related to possible anomalies) which we are not going to focus on here, the simultaneous kernel of $\Theta$ and $d \Theta$ is spanned by $\left\langle\tilde{X}_{l^{\mu}(\mathbf{0})}^{L}, \tilde{X}_{s^{\mu}(\mathbf{0})}^{L}, \tilde{X}_{a(\mathbf{0})}^{L}\right\rangle$, and denoted by $\mathcal{G}_{\Theta}$. These vector fields are the generators of the generalized equations of motion, the classical phase space being given by $G / G_{\Theta}$. The Noether invariants under these classical trajectories are

$$
\begin{aligned}
& \mathcal{N}_{l^{\tau}(\mathbf{t})}=i_{\tilde{X}_{l^{\tau}(\mathbf{t})}^{R}} \Theta \\
& =C_{\tau(\mathbf{t}) \mu(\mathbf{m})}^{\varphi} l^{\mu}(\mathbf{m})+C_{\tau(\mathbf{t}) \tilde{\mu}(\mathbf{m})}^{\varphi} s^{\mu}(\mathbf{m})+C_{\tau(\mathbf{t}) \mathbf{m}}^{\varphi} a(\mathbf{m}) \\
& +\frac{3}{4}\left(\frac{1}{2} C_{\rho(\mathbf{r}) \mu(\mathbf{m})}^{\varphi} C_{\sigma(\mathbf{s}) \tau(\mathbf{t})}^{\mu(\mathbf{m})}+C_{\rho(\mathbf{r}) \tilde{\mu}(\mathbf{m})}^{\varphi} C_{\gamma(\mathbf{q}) \tau(\mathbf{t})}^{\tilde{\mu}(\mathbf{m})}\right) l^{\rho}(\mathbf{r}) l^{\gamma}(\mathbf{q}) \\
& +\quad C_{\rho(\mathbf{r}) \tilde{\mu}(\mathbf{m})}^{\varphi} C_{\tilde{\gamma}(\mathbf{q}) \tau(\mathbf{t})}^{\tilde{\mu}(\mathbf{m})} l^{\rho}(\mathbf{r}) s^{\gamma}(\mathbf{q})+\frac{1}{2} C_{\mathbf{r m}}^{\varphi} C_{\mathbf{q} \tau(\mathbf{t})}^{\mathbf{m}} a(\mathbf{r}) a(\mathbf{q})+C_{\rho(\mathbf{r}) \mathbf{m}}^{\varphi} C_{\mathbf{q} \tau(\mathbf{t})}^{\mathbf{m}} l^{\rho}(\mathbf{r}) a(\mathbf{q})+\ldots \\
& \mathcal{N}_{s^{\tau}(\mathbf{t})}=i_{\tilde{X}_{s^{\tau}(\mathbf{t})}^{R}} \Theta \\
& =C_{\tilde{\tau}(\mathbf{t}) \mu(\mathbf{m})}^{\varphi} l^{\mu}(\mathbf{m})+\frac{1}{2} C_{\rho(\mathbf{r}) \tilde{\mu}(\mathbf{m})}^{\varphi} C_{\gamma(\mathbf{q}) \tilde{\tau}(\mathbf{t})}^{\tilde{\mu}(\mathbf{m})} l^{\rho}(\mathbf{r}) l^{\gamma}(\mathbf{q})+\ldots \\
& \mathcal{N}_{a(\mathbf{t})}=i_{\tilde{X}_{a(\mathbf{t})}^{R}} \Theta \\
& =C_{\mathbf{t m}}^{\varphi} a(\mathbf{m})+C_{\mathbf{t} \mu(\mathbf{m})}^{\varphi} l^{\mu}(\mathbf{m}) \\
& +\frac{1}{4}\left(2 C_{\mathbf{m t}}^{\varphi} C_{\rho(\mathbf{r}) \mathbf{q}}^{\mathbf{m}}+C_{\mathbf{q} \mathbf{m}}^{\varphi} C_{\rho(\mathbf{r}) \mathbf{t}}^{\mathbf{m}}+2 C_{\rho(\mathbf{r}) \tilde{\mu}(\mathbf{m})}^{\varphi} C_{\mathbf{q} \mathbf{t}}^{\tilde{\mu}(\mathbf{m})}\right) l^{\rho}(\mathbf{r}) a(\mathbf{q}) \\
& +\frac{1}{2} C_{\rho(\mathbf{r}) \mathbf{m}}^{\varphi} C_{\gamma(\mathbf{q}) \mathbf{t}}^{\mathbf{m}} l^{\rho}(\mathbf{r}) l^{\gamma}(\mathbf{q})+\ldots .
\end{aligned}
$$


Finally, the Noether invariants associated with the vector fields inside $\mathcal{G}_{\Theta}$ can be written in terms of the remaining (basic) Noether invariants, which can be used as coordinates in the solution manifold. Up to the second order (inherited from the third order in the group law) we find,

$$
\begin{aligned}
& \mathcal{N}_{l^{\tau}(\mathbf{0})}=\left\{\frac{3}{4}\left(\frac{1}{2} C_{\rho(\mathbf{r}) \mu(\mathbf{m})}^{\varphi} C_{\gamma(\mathbf{q}) \tau(\mathbf{0})}^{\mu(\mathbf{m})}+C_{\rho(\mathbf{r}) \tilde{\mu}(\mathbf{m})}^{\varphi} C_{\gamma(\mathbf{q}) \tau(\mathbf{0})}^{\tilde{\mu}(\mathbf{m})}\right)\left(C_{\tilde{\nu}(\mathbf{n}) \rho(\mathbf{r})}^{\varphi}\right)^{-1}\left(C_{\tilde{\nu}^{\prime}\left(\mathbf{n}^{\prime}\right) \gamma(\mathbf{q})}^{\varphi}\right)^{-1}\right. \\
& -\quad C_{\rho(\mathbf{r}) \tilde{\mu}(\mathbf{m})}^{\varphi} C_{\tilde{\gamma}(\mathbf{q}) \tau(\mathbf{0})}^{\tilde{\mu}(\mathbf{m})}\left(C_{\tilde{\nu}(\mathbf{n}) \rho(\mathbf{r})}^{\varphi}\right)^{-1}\left(C_{\alpha(\mathbf{a}) \tilde{\gamma}(\mathbf{q})}^{\varphi}\right)^{-1}\left(C_{\alpha(\mathbf{a}) \gamma(\mathbf{q})}^{\varphi}\left(C_{\tilde{\nu}^{\prime}\left(\mathbf{n}^{\prime}\right) \gamma^{\prime}\left(\mathbf{q}^{\prime}\right)}^{\varphi}\right)^{-1}\right. \\
& \left.-C_{\alpha(\mathbf{a}) \mathbf{q}^{\prime}}^{\varphi}\left(C_{\mathbf{a}^{\prime} \mathbf{q}^{\prime}}^{\varphi}\right)^{-1} C_{\mathbf{a}^{\prime} \rho^{\prime}\left(\mathbf{r}^{\prime}\right)}^{\varphi}\left(C_{\tilde{\nu}^{\prime}\left(\mathbf{n}^{\prime}\right) \rho^{\prime}\left(\mathbf{r}^{\prime}\right)}^{\varphi}\right)^{-1}\right) \\
& +\frac{1}{2} C_{\mathbf{r m}}^{\varphi} C_{\mathbf{s} \tau(\mathbf{0})}^{\mathbf{m}}\left(C_{\mathbf{t}^{\prime} \mathbf{r}}^{\varphi}\right)^{-1} C_{\mathbf{t}^{\prime} \gamma^{\prime}\left(\mathbf{q}^{\prime}\right)}^{\varphi}\left(C_{\tilde{\nu}(\mathbf{n}) \gamma^{\prime}\left(\mathbf{q}^{\prime}\right)}^{\varphi}\right)^{-1}\left(C_{\mathbf{t}^{\prime \prime} \mathbf{s}}^{\varphi}\right)^{-1} C_{\mathbf{t}^{\prime \prime} \gamma^{\prime \prime}\left(\mathbf{q}^{\prime \prime}\right)}^{\varphi}\left(C_{\tilde{\nu}^{\prime}\left(\mathbf{n}^{\prime}\right) \gamma^{\prime \prime}\left(\mathbf{q}^{\prime \prime}\right)}^{\varphi}\right)^{-1} \\
& \text { - } \left.C_{\rho(\mathbf{r}) \mu}^{\varphi} C_{\mathbf{s} \tau(\mathbf{0})}^{\mu}\left(C_{\tilde{\nu}(\mathbf{n}) \rho(\mathbf{r})}^{\varphi}\right)^{-1}\left(C_{\mathbf{t}^{\prime \prime} \mathbf{s}}^{\varphi}\right)^{-1} C_{\mathbf{t}^{\prime \prime} \gamma^{\prime \prime}\left(\mathbf{q}^{\prime \prime}\right)}^{\varphi}\left(C_{\tilde{\nu}^{\prime}\left(\mathbf{n}^{\prime}\right) \gamma^{\prime \prime}\left(\mathbf{q}^{\prime \prime}\right)}^{\varphi}\right)^{-1}\right\} \mathcal{N}_{s^{\nu}(\mathbf{n})} \mathcal{N}_{s^{\nu^{\prime}}\left(\mathbf{n}^{\prime}\right)} \\
& +\quad C_{\rho(\mathbf{r}) \tilde{\mu}(\mathbf{m})}^{\varphi} C_{\tilde{\gamma}(\mathbf{q}) \tau(\mathbf{0})}^{\tilde{\mu}(\mathbf{m})}\left(C_{\tilde{\nu}(\mathbf{n}) \rho(\mathbf{r})}^{\varphi}\right)^{-1}\left(C_{\alpha(\mathbf{a}) \tilde{\gamma}(\mathbf{q})}^{\varphi}\right)^{-1} \mathcal{N}_{s^{\nu}(\mathbf{n})} \mathcal{N}_{l^{\alpha}(\mathbf{a})} \\
& +\left\{-C_{\rho(\mathbf{r}) \tilde{\mu}(\mathbf{m})}^{\varphi} C_{\tilde{\gamma}(\mathbf{q}) \tau(\mathbf{0})}^{\tilde{\mu}(\mathbf{m})}\left(C_{\tilde{\nu}(\mathbf{n}) \rho(\mathbf{r})}^{\varphi}\right)^{-1}\left(C_{\alpha(\mathbf{a}) \tilde{\gamma}(\mathbf{q})}^{\varphi}\right)^{-1} C_{\alpha(\mathbf{a}) \mathbf{q}^{\prime}}^{\varphi}\left(C_{\mathbf{n}^{\prime} \mathbf{q}^{\prime}}^{\varphi}\right)^{-1}\right. \\
& -\frac{1}{2} C_{\mathbf{r m}}^{\varphi} C_{\mathbf{s} \tau(\mathbf{0})}^{\mathbf{m}}\left(C_{\mathbf{n}^{\prime} \mathbf{r}}^{\varphi}\right)^{-1}\left(C_{\mathbf{t}^{\prime \prime} \mathbf{s}}^{\varphi}\right)^{-1} C_{\mathbf{t}^{\prime \prime} \gamma^{\prime \prime}\left(\mathbf{q}^{\prime \prime}\right)}^{\varphi}\left(C_{\tilde{\nu}(\mathbf{n}) \gamma^{\prime \prime}\left(\mathbf{q}^{\prime \prime}\right)}^{\varphi}\right)^{-1} \\
& \left.+\left(C_{\mathbf{n}^{\prime} \mathbf{s}}^{\varphi}\right)^{-1} C_{\mathbf{s} \gamma^{\prime}\left(\mathbf{q}^{\prime}\right)}^{\varphi}\left(C_{\tilde{\nu}(\mathbf{n}) \gamma^{\prime}\left(\mathbf{q}^{\prime}\right)}^{\varphi}\right)^{-1}+C_{\rho(\mathbf{r}) \mu}^{\varphi} C_{\mathbf{s} \tau(\mathbf{0})}^{\mu}\left(C_{\tilde{\nu}(\mathbf{n}) \rho(\mathbf{r})}^{\varphi}\right)^{-1}\left(C_{\mathbf{n}^{\prime} \mathbf{s}}^{\varphi}\right)^{-1}\right\} \\
& \text {. } \quad \mathcal{N}_{s^{\nu}(\mathbf{n})} \mathcal{N}_{a\left(\mathbf{n}^{\prime}\right)}+\frac{1}{2} C_{\mathbf{r m}}^{\varphi} C_{\mathbf{q} \tau(\mathbf{0})}^{\mathbf{m}}\left(C_{\mathbf{t}^{\prime} \mathbf{q}}^{\varphi}\right)^{-1}\left(C_{\mathbf{t}^{\prime \prime} \mathbf{r}}^{\varphi}\right)^{-1} \mathcal{N}_{a\left(\mathbf{t}^{\prime}\right)} \mathcal{N}_{a\left(\mathbf{t}^{\prime \prime}\right)}+\ldots \\
& \mathcal{N}_{s^{\tau}(\mathbf{0})}=\frac{1}{2} C_{\rho(\mathbf{r}) \tilde{\mu}(\mathbf{m})}^{\varphi} C_{\gamma(\mathbf{q}) \tilde{\tau}(\mathbf{0})}^{\tilde{\mu}(\mathbf{m})}\left(C_{\tilde{\tau}^{\prime}\left(\mathbf{t}^{\prime}\right) \rho(\mathbf{r})}^{\varphi}\right)^{-1}\left(C_{\tilde{\tau}^{\prime \prime}\left(\mathbf{t}^{\prime \prime}\right) \gamma(\mathbf{q})}^{\varphi}\right)^{-1} \mathcal{N}_{s^{\tau^{\prime}}\left(\mathbf{t}^{\prime}\right)} \mathcal{N}_{s^{\tau^{\prime \prime}}\left(\mathbf{t}^{\prime \prime}\right)}+\ldots \\
& \mathcal{N}_{a(\mathbf{0})}=\left\{-\frac{1}{4}\left(2 C_{\mathbf{m} \mathbf{0}}^{\varphi} C_{\rho(\mathbf{r}) \mathbf{q}}^{\mathbf{m}}+C_{\mathbf{q} \mathbf{m}}^{\varphi} C_{\rho(\mathbf{r}) \mathbf{0}}^{\mathbf{m}}+2 C_{\rho(\mathbf{r}) \tilde{\mu}(\mathbf{m})}^{\varphi} C_{\mathbf{q} \mathbf{0}}^{\tilde{\mu}(\mathbf{m})}\right)\right. \\
& \left(C_{\tilde{\tau}^{\prime}\left(\mathbf{t}^{\prime}\right) \rho(\mathbf{r})}^{\varphi}\right)^{-1}\left(C_{\mathbf{t}^{\prime \prime} \mathbf{q}}^{\varphi}\right)^{-1} C_{\mathbf{t}^{\prime \prime} \gamma^{\prime \prime}\left(\mathbf{q}^{\prime \prime}\right)}^{\varphi}\left(C_{\tilde{\nu}^{\prime \prime}\left(\mathbf{n}^{\prime \prime}\right) \gamma^{\prime \prime}\left(\mathbf{q}^{\prime \prime}\right)}^{\varphi}\right)^{-1} \\
& \left.+\frac{1}{2} C_{\rho(\mathbf{r}) \mathbf{m}}^{\varphi} C_{\gamma(\mathbf{q}) \mathbf{0}}^{\mathbf{m}}\left(C_{\tilde{\tau}^{\prime}\left(\mathbf{t}^{\prime}\right) \rho(\mathbf{r})}^{\varphi}\right)^{-1}\left(C_{\tilde{\nu}^{\prime \prime}\left(\mathbf{n}^{\prime \prime}\right) \gamma(\mathbf{q})}^{\varphi}\right)^{-1}\right\} \mathcal{N}_{s^{\tau^{\prime}}\left(\mathbf{t}^{\prime}\right)} \mathcal{N}_{s^{\nu^{\prime \prime}}\left(\mathbf{n}^{\prime \prime}\right)} \\
& +\left\{\frac{1}{4}\left(2 C_{\mathbf{m} \mathbf{0}}^{\varphi} C_{\rho(\mathbf{r}) \mathbf{q}}^{\mathbf{m}}+C_{\mathbf{q} \mathbf{m}}^{\varphi} C_{\rho(\mathbf{r}) \mathbf{0}}^{\mathbf{m}}+2 C_{\rho(\mathbf{r}) \tilde{\mu}(\mathbf{m})}^{\varphi} C_{\mathbf{q} \mathbf{0}}^{\tilde{\mu}(\mathbf{m})}\right)\left(C_{\tilde{\tau}^{\prime}\left(\mathbf{t}^{\prime}\right) \rho(\mathbf{r})}^{\varphi}\right)^{-1}\left(C_{\mathbf{t}^{\prime \prime} \mathbf{q}}^{\varphi}\right)^{-1}\right\} \\
& \cdot \mathcal{N}_{s^{\tau^{\prime}}\left(\mathbf{t}^{\prime}\right)} \mathcal{N}_{a\left(\mathbf{t}^{\prime \prime}\right)}+\ldots
\end{aligned}
$$

\subsection{Quantum analysis}

The regular representation is originally realized on those complex functions defined on the extended group which satisfy the $U(1)$-equivariance condition

$$
\tilde{X}_{\varphi}^{R} \Psi=\frac{\partial}{\partial \varphi} \Psi=i \Psi
$$

and is infinitesimally implemented by the right-invariant vector fields. In order to reduce this representation, we must impose a polarization condition associated with a (left-invariant) polarization algebra $\mathcal{G}_{\mathcal{P}}$, implying the selection of a particular class of representations.

We shall implement the easiest polarization one can conceive in this context. In order to do so, we introduce the following notation: given a vector $\mathbf{m} \neq 0$, let us call $m_{f}$ the first non-zero 
entry $m_{i}$. For $\mathbf{m}=0$, let $m_{f}=0$. Now we introduce the following polarization:

$$
\mathcal{G}_{\Theta}=\left\langle X_{l^{\mu}(\mathbf{t})_{\left(t_{f} \leq 0\right)}^{L}}^{L}, X_{s^{\mu}(\mathbf{t})_{\left(t_{f} \leq 0\right)}^{L}}^{L}, X_{a(\mathbf{t})_{\left(t_{f} \leq 0\right)}^{L}}^{L}\right\rangle,
$$

defining the polarization equations:

$$
\begin{aligned}
& X_{l^{\mu}(\mathbf{t})_{\left(t_{f} \leq 0\right)}^{L}} \Psi=0
\end{aligned}
$$

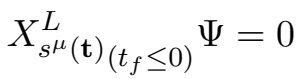

$$
\begin{aligned}
& X_{a(\mathbf{t})\left(t_{f} \leq 0\right)}^{L} \Psi=0 .
\end{aligned}
$$

A general solution can be written as a linear combination:

$$
\begin{aligned}
& \Psi=A|0\rangle+\sum_{\mathbf{m}\left(m_{f}>0\right)}\left(A_{\mu(\mathbf{m})}\left|l^{\mu}(\mathbf{m})\right\rangle+A_{\tilde{\mu}(\mathbf{m})}\left|s^{\mu}(\mathbf{m})\right\rangle+A_{\mathbf{m}}|a(\mathbf{m})\rangle\right) \\
& +\sum\left(A_{\mu(\mathbf{m}) \nu(\mathbf{n})}\left|l^{\mu}(\mathbf{m}) l^{\nu}(\mathbf{n})\right\rangle+A_{\mu(\mathbf{m}) \tilde{\nu}(\mathbf{n})}\left|l^{\mu}(\mathbf{m}) s^{\nu}(\mathbf{n})\right\rangle\right. \\
& +A_{\mu(\mathbf{m}) \mathbf{n}}\left|l^{\mu}(\mathbf{m}) a(\mathbf{n})\right\rangle+A_{\tilde{\mu}(\mathbf{m}) \tilde{\nu}(\mathbf{n})}\left|s^{\mu}(\mathbf{m}) s^{\nu}(\mathbf{n})\right\rangle \\
& \left.+A_{\tilde{\mu}(\mathbf{m}) \mathbf{n}}\left|s^{\mu}(\mathbf{m}) a(\mathbf{n})\right\rangle+A_{\mathbf{m n}}|a(\mathbf{m}) a(\mathbf{n})\rangle\right),
\end{aligned}
$$

where the functions in the basis, $|\ldots\rangle$, are already polarized and present in fact a product structure in terms of the functions $\{\ldots\}$ that we are giving below; they share a common weight function $W$ depending on the representation,

$$
\begin{aligned}
|0\rangle & =\zeta W \\
\left|l^{\mu}(\mathbf{m})\right\rangle=\zeta W\left\{l^{\mu}(\mathbf{m})\right\}, \quad\left|s^{\mu}(\mathbf{m})\right\rangle & =\zeta W\left\{s^{\mu}(\mathbf{m})\right\}, \quad|a(\mathbf{m})\rangle=\zeta W\{a(\mathbf{m})\} \\
\left|l^{\mu_{1}}\left(\mathbf{m}_{i}\right) \ldots s^{\mu_{1}}\left(\mathbf{m}_{j}\right) \ldots a\left(\mathbf{m}_{k}\right) \ldots\right\rangle & =\zeta W\left\{l^{\mu_{1}}\left(\mathbf{m}_{i}\right)\right\} \ldots\left\{s^{\mu_{1}}\left(\mathbf{m}_{j}\right)\right\} \ldots\left\{a\left(\mathbf{m}_{k}\right)\right\} \ldots .
\end{aligned}
$$

The explicit forms of the corresponding functions are,

$$
\begin{aligned}
W & =1-\sum_{\mathbf{n}_{\left(n_{f}>0\right)}}\left(\frac{1}{2} C_{\nu(\mathbf{n}) \mu(\mathbf{m})}^{\varphi} l^{\mu}(\mathbf{m}) l^{\nu}(\mathbf{n})+\frac{1}{2} C_{\mathbf{m} \mathbf{n}}^{\varphi} a(\mathbf{m}) a(\mathbf{n})\right. \\
& \left.+C_{\mu(\mathbf{m}) \tilde{\nu}(\mathbf{n})}^{\varphi} l^{\mu}(\mathbf{m}) s^{\nu}(\mathbf{n})+C_{\mu(\mathbf{m}) \mathbf{n}}^{\varphi} l^{\mu}(\mathbf{m}) a(\mathbf{n})+\ldots\right) \\
\left\{l^{\gamma}(\mathbf{q})\right\} & =l^{\gamma}(\mathbf{q})-\sum_{t_{f} \leq 0, m_{f}>0, n_{f}>0, m_{f}+n_{f} \geq q_{f}}\left[\frac{1}{2} C_{\mu(\mathbf{m}) \tau(\mathbf{t})}^{\gamma(\mathbf{q})} l^{\mu}(\mathbf{m}) l^{\tau}(\mathbf{t})\right. \\
& +\frac{1}{8}\left(-\sum_{r_{f} \leq 0} C_{\mu(\mathbf{m}) \tau(\mathbf{t})}^{\rho(\mathbf{r})} C_{\nu(\mathbf{n}) \rho(\mathbf{r})}^{\gamma(\mathbf{q})}+\sum_{r_{f}>0}\left(C_{\rho(\mathbf{r}) \tau(\mathbf{t})}^{\gamma(\mathbf{q})} C_{\mu(\mathbf{m}) \nu(\mathbf{n})}^{\rho(\mathbf{r})}\right.\right. \\
& \left.\left.+C_{\mu(\mathbf{m}) \rho(\mathbf{r})}^{\gamma(\mathbf{q})} C_{\nu(\mathbf{n}) \tau(\mathbf{t})}^{\rho(\mathbf{r})}\right) l^{\tau}(\mathbf{t}) l^{\mu}(\mathbf{m}) l^{\nu}(\mathbf{n})+\ldots\right] \\
\left\{s^{\gamma}(\mathbf{q})\right\} & =s^{\gamma}(\mathbf{q})-\sum_{t_{f} \leq 0, m_{f}>0, n_{f}>0, m_{f}+n_{f} \geq q_{f}}\left(\frac{1}{2} C_{\mu(\mathbf{m}) \tau(\mathbf{t})}^{\tilde{\gamma}(\mathbf{q})} l^{\mu}(\mathbf{m}) l^{\tau}(\mathbf{t})+C_{\mu(\mathbf{m}) \tilde{\tau}(\mathbf{t})} l^{\mu}(\mathbf{m}) s^{\tau}(\mathbf{t})\right. \\
& \left.\left.+\frac{1}{2} C_{\mathbf{m t}}^{\tilde{\gamma}(\mathbf{q})}\right) a(\mathbf{m}) a(\mathbf{t})\right)+\sum_{r_{f} \leq 0}\left(\frac{1}{8} C_{\mu(\mathbf{m}) \tau(\mathbf{t})}^{\rho(\mathbf{r})} C_{\nu(\mathbf{n}) \rho(\mathbf{r})}^{\tilde{\gamma}(\mathbf{q})}-\frac{1}{2} C_{\mu(\mathbf{m}) \tau(\mathbf{t})}^{\tilde{\rho}(\mathbf{r})} C_{\nu(\mathbf{n}) \tilde{\rho}(\mathbf{r})}^{\tilde{\gamma}(\mathbf{q})}\right)
\end{aligned}
$$




$$
\begin{aligned}
+ & \left.\sum_{r_{f}>0}\left(-\frac{1}{8} C_{\rho(\mathbf{r}) \tau(\mathbf{t})}^{\tilde{\gamma}(\mathbf{(})} C_{\mu(\mathbf{m}) \nu(\mathbf{n})}^{\rho(\mathbf{r})}+\frac{1}{4} C_{\mu(\mathbf{m}) \rho(\mathbf{r})}^{\tilde{\gamma}(\mathbf{q})} C_{\nu(\mathbf{n}) \tau(\mathbf{t})}^{\rho(\mathbf{r})}\right)\right] l^{\tau}(\mathbf{t}) l^{\mu}(\mathbf{m}) l^{\nu}(\mathbf{n}) \\
+ & \frac{1}{2}\left[\sum_{r_{f} \leq 0} C_{\mu(\mathbf{m}) \tilde{\tau}(\mathbf{t})}^{\tilde{\rho}(\mathbf{r})} C_{\nu(\mathbf{n}) \tilde{\rho}(\mathbf{r})}^{\tilde{\gamma}(\mathbf{q})}+\sum_{r_{f}>0}\left(\frac{1}{2} C_{\rho(\mathbf{r}) \tilde{\tau}(\mathbf{t})}^{\tilde{\gamma}(\mathbf{(})} C_{\mu(\mathbf{m}) \nu(\mathbf{n})}^{\rho(\mathbf{r})}\right.\right. \\
+ & \left.\left.C_{\mu(\mathbf{m}) \tilde{\rho}(\mathbf{r})}^{\tilde{\gamma}(\mathbf{q})} C_{\nu(\mathbf{n}) \tilde{\tau}(\mathbf{t})}^{\tilde{\rho}(\mathbf{r})}\right)\right] l^{\mu}(\mathbf{m}) l^{\nu}(\mathbf{n}) s^{\tau}(\mathbf{t})+ \\
& -\frac{1}{4}\left[\sum_{r_{f} \leq 0}\left(C_{\mu(\mathbf{m}) \mathbf{t}}^{\mathbf{r}} C_{\mathbf{n r}}^{\tilde{\gamma}(\mathbf{q})}+2 C_{\mathbf{n t}}^{\tilde{\rho}(\mathbf{r})} C_{\mu(\mathbf{m}) \tilde{\rho}(\mathbf{r})}^{\tilde{\gamma}(\mathbf{q})}\right)-\sum_{r_{f}>0} C_{\mathbf{n} \mathbf{r}}^{\tilde{\gamma}(\mathbf{q})} C_{\mu(\mathbf{m}) \mathbf{t}}^{\mathbf{r}}\right] l^{\mu}(\mathbf{m}) a(\mathbf{t}) a(\mathbf{n})+\ldots \\
\{a(\mathbf{q})\} & =a(\mathbf{q})- \\
& \sum_{t_{f} \leq 0, m_{f}>0, n_{f}>0, m_{f}+n_{f} \geq q_{f}}\left(C_{\mu(\mathbf{m}) \mathbf{t}}^{\mathbf{q}} l^{\mu(\mathbf{m})} a(\mathbf{t})-\frac{1}{2}\left[\sum_{r_{f} \leq 0} C_{\mu(\mathbf{m}) \mathbf{t}}^{\mathbf{r}} C_{\nu(\mathbf{n}) \mathbf{r}}^{\mathbf{q}}\right.\right. \\
+ & \left.\left.\sum_{r_{f}>0}\left(\frac{1}{2} C_{\rho(\mathbf{r}) \mathbf{t}}^{\mathbf{q}} C_{\mu(\mathbf{m}) \nu(\mathbf{n})}^{\rho(\mathbf{r})}-C_{\mu(\mathbf{m}) \mathbf{r}}^{\mathbf{q}} C_{\nu(\mathbf{n}) \mathbf{t}}^{\mathbf{r}}\right)\right] l^{\mu}(\mathbf{m}) l^{\nu(\mathbf{n})} a(\mathbf{t})+\ldots\right) .
\end{aligned}
$$

The action of the starting algebra is implemented by the right-invariant vector fields in such a way that those vector fields with $m_{f} \leq 0$ act as raising operators and those with $m_{f}>0$ are lowering operators. Finally, the explicit action of these right-invariant vector fields on the lowest-order functions of the reduced Hilbert space is:

$$
\begin{aligned}
& \tilde{X}_{a(\mathbf{t})_{\left(t_{f} \leq 0\right)}^{R}}^{R}|0\rangle=C_{\mathbf{t n}}^{\varphi}|a(\mathbf{n})\rangle+C_{\mathbf{t} \nu(\mathbf{n})}^{\varphi}\left|l^{\nu(\mathbf{n})}\right\rangle-\frac{1}{2} \sum_{r_{f} \leq 0} C_{\mathbf{t n}}^{\tilde{\rho}(\mathbf{r})} C_{\mu(\mathbf{m}) \tilde{\rho}(\mathbf{r})}^{\varphi}\left|a(\mathbf{n}) l^{\mu}(\mathbf{m})\right\rangle+\ldots \\
& \tilde{X}_{a(\mathbf{t})_{\left(t_{f} \leq 0\right)}^{R}}^{R}\left|l^{\gamma}(\mathbf{q})\right\rangle=C_{\mathbf{t n}}^{\varphi}\left|a(\mathbf{n}) l^{\gamma}(\mathbf{q})\right\rangle+C_{\mathbf{t} \nu(\mathbf{n})}^{\varphi}\left|l^{\nu}(\mathbf{n}) l^{\gamma}(\mathbf{q})\right\rangle+\ldots \\
& \tilde{X}_{a(\mathbf{t})_{\left(t_{f} \leq 0\right)}^{R}}^{R}\left|s^{\gamma}(\mathbf{q})\right\rangle=C_{\mathbf{t n}}^{\tilde{\gamma}(\mathbf{q})}|a(\mathbf{n})\rangle+C_{\mathbf{t n}}^{\varphi}\left|a(\mathbf{n}) s^{\gamma}(\mathbf{q})\right\rangle+C_{\mathbf{t} \nu(\mathbf{n})}^{\varphi}\left|l^{\nu}(\mathbf{n}) s^{\gamma}(\mathbf{q})\right\rangle \\
& +\left[\sum_{r_{f} \leq 0}\left(\frac{1}{4} C_{\mu(\mathbf{m}) \mathbf{t}}^{\mathbf{r}} C_{\mathbf{n r}}^{\tilde{\gamma}(\mathbf{q})}+C_{\mathbf{n t}}^{\tilde{\rho}(\mathbf{r})} C_{\mu(\mathbf{m}) \tilde{\rho}(\mathbf{r})}^{\tilde{\gamma}(\mathbf{q})}\right)\right. \\
& \left.\left.-\sum_{r_{f}>0} \frac{1}{4} C_{\mu(\mathbf{m}) \mathbf{t}}^{\mathbf{r}} C_{\mathbf{n r}}^{\tilde{\gamma}(\mathbf{q})}\right)\right]\left|l^{\mu}(\mathbf{m}) a(\mathbf{n})\right\rangle+\ldots \\
& \tilde{X}_{a(\mathbf{t})_{\left(t_{f} \leq 0\right)}^{R}}^{R}|a(\mathbf{q})\rangle=C_{\mathbf{t n}}^{\varphi}|a(\mathbf{n}) a(\mathbf{q})\rangle+C_{\mathbf{t} \nu(\mathbf{n})}^{\varphi}\left|l^{\nu}(\mathbf{n}) a(\mathbf{q})\right\rangle+\frac{1}{2} C_{\mathbf{t} \mu(\mathbf{m})}^{\mathbf{q}}\left|l^{\mu}(\mathbf{m})\right\rangle \\
& -\frac{1}{2}\left[\sum_{r_{f} \leq 0} C_{\mathbf{t} \mu(\mathbf{m})}^{\mathbf{r}} C_{\nu(\mathbf{n}) \mathbf{r}}^{\mathbf{q}}+\sum_{r_{f}>0} C_{\mu(\mathbf{m}) \mathbf{r}}^{\mathbf{q}} C_{\nu(\mathbf{n}) \mathbf{t}}^{\mathbf{r}}\right. \\
& \left.-\quad \frac{1}{2} C_{\rho(\mathbf{r}) \mathbf{t}}^{\mathbf{q}} C_{\mu(\mathbf{m}) \nu(\mathbf{n})}^{\rho(\mathbf{r})}\right]\left|l^{\mu}(\mathbf{m}) l^{\nu}(\mathbf{n})\right\rangle+\ldots \\
& \tilde{X}_{s^{\tau}(\mathbf{t})_{\left(t_{f} \leq 0\right)}^{R}}^{R}|0\rangle=C_{\tilde{\tau}(\mathbf{t}) \nu(\mathbf{n})}^{\varphi}\left|l^{\nu}(\mathbf{n})\right\rangle+\ldots \\
& \tilde{X}_{s^{\tau}(\mathbf{t})_{\left(t_{f} \leq 0\right)}^{R}}^{R}\left|l^{\gamma}(\mathbf{q})\right\rangle=C \varphi_{\tilde{\tau}(\mathbf{t}) \nu(\mathbf{n})}\left|l^{\nu}(\mathbf{n}) l^{\gamma}(\mathbf{q})\right\rangle+\ldots
\end{aligned}
$$




$$
\begin{aligned}
& \tilde{X}_{s^{\tau}(\mathbf{t})_{\left(t_{f} \leq 0\right)}^{R}}^{R}\left|s^{\gamma}(\mathbf{q})\right\rangle=C_{\tilde{\tau}(\mathbf{t}) \mu(\mathbf{m})}^{\tilde{\gamma}(\mathbf{q})}\left|l^{\mu}(\mathbf{m})\right\rangle+C_{\tilde{\tau}(\mathbf{t}) \nu(\mathbf{n})}^{\varphi}\left|l^{\nu}(\mathbf{n}) s^{\gamma}(\mathbf{q})\right\rangle \\
& +\frac{1}{2}\left[\sum_{r_{f} \leq 0} C_{\mu(\mathbf{m}) \tilde{\tau}(\mathbf{t})}^{\tilde{\rho}(\mathbf{r})} C_{\nu(\mathbf{n}) \tilde{\rho}(\mathbf{r})}^{\tilde{\gamma}(\mathbf{( r )}}+\sum_{r_{f}>0}\left(\frac{1}{2} C_{\rho(\mathbf{r}) \tilde{\tau}(\mathbf{t})}^{\tilde{\gamma}(\mathbf{(})} C_{\mu(\mathbf{m}) \nu(\mathbf{n})}^{\rho(\mathbf{r})}\right.\right. \\
& \left.\left.-\quad C_{\mu(\mathbf{m}) \tilde{\rho}(\mathbf{r})}^{\tilde{\gamma}(\mathbf{(})} C_{\nu(\mathbf{n}) \tilde{\tau}(\mathbf{t})}^{\tilde{\rho}(\mathbf{r})}\right)\right]\left|l^{\mu}(\mathbf{m}) l^{\nu}(\mathbf{n})\right\rangle+\ldots \\
& \tilde{X}_{s^{\tau}(\mathbf{t})_{\left(t_{f} \leq 0\right)}^{R}}^{R}|a(\mathbf{q})\rangle=C_{\tilde{\tau}(\mathbf{t}) \nu(\mathbf{n})}^{\varphi}\left|l^{\nu}(\mathbf{n}) a(\mathbf{q})\right\rangle+\ldots \\
& \tilde{X}_{l^{\tau}(\mathbf{t})_{\left(t_{f} \leq 0\right)}}^{R}|0\rangle=C_{\tau(\mathbf{t}) \nu(\mathbf{n})}^{\varphi}\left|l^{\nu}(\mathbf{n})\right\rangle+C_{\tau(\mathbf{t}) \tilde{\nu}(\mathbf{n})}^{\varphi}\left|s^{\nu}(\mathbf{n})\right\rangle+C_{\tau(\mathbf{t}) \mathbf{n}}^{\varphi}|a(\mathbf{n})\rangle \\
& -\sum_{r_{f} \leq 0}\left(\frac{1}{4} C_{\tau(\mathbf{t}) \nu(\mathbf{n})}^{\rho(\mathbf{r})} C_{\mu(\mathbf{m}) \rho(\mathbf{r})}^{\varphi}+\frac{1}{2} C_{\tau(\mathbf{t}) \nu(\mathbf{n})}^{\tilde{\rho}(\mathbf{r})} C_{\mu(\mathbf{m}) \tilde{\rho}(\mathbf{r})}^{\varphi}\right)\left|l^{\nu}(\mathbf{n}) l^{\mu}(\mathbf{m})\right\rangle \\
& -\sum_{r_{f} \leq 0} C_{\tau(\mathbf{t}) \tilde{\nu}(\mathbf{n})}^{\tilde{\rho}(\mathbf{r})} C_{\mu(\mathbf{m}) \tilde{\rho}(\mathbf{r})}^{\varphi}\left|s^{\nu}(\mathbf{n}) l^{\mu}(\mathbf{m})\right\rangle-\frac{1}{2} \sum_{r_{f} \leq 0} C_{\tau(\mathbf{t}) \mathbf{n}}^{\mathbf{r}} C_{\mathbf{m r}}^{\varphi}|a(\mathbf{n}) a(\mathbf{m})\rangle \\
& -\sum_{r_{f} \leq 0} C_{\tau(\mathbf{t}) \mathbf{n}}^{\mathbf{r}} C_{\mu(\mathbf{m}) \mathbf{r}}^{\varphi}\left|a(\mathbf{n}) l^{\mu}(\mathbf{m})\right\rangle+\ldots \\
& \tilde{X}_{l^{\tau}(\mathbf{t})_{\left(t_{f} \leq 0\right)}}^{R}\left|l^{\gamma}(\mathbf{q})\right\rangle=C_{\tau(\mathbf{t}) \mu(\mathbf{m})}^{\gamma(\mathbf{q})}\left|l^{\mu}(\mathbf{m})\right\rangle+C_{\tau(\mathbf{t}) \nu(\mathbf{n})}^{\varphi}\left|l^{\nu}(\mathbf{n}) l^{\gamma}(\mathbf{q})\right\rangle+C_{\tau(\mathbf{t}) \tilde{\nu}(\mathbf{n})}^{\varphi}\left|s^{\nu}(\mathbf{n}) l^{\gamma}(\mathbf{q})\right\rangle \\
& +C_{\tau(\mathbf{t}) \mathbf{n}}^{\varphi}\left|a(\mathbf{n}) l^{\gamma}(\mathbf{q})\right\rangle-\frac{1}{8}\left[3 \sum_{r_{f} \leq 0} C_{\tau(\mathbf{t}) \nu(\mathbf{n}}^{\rho(\mathbf{r})}\right) C_{\mu(\mathbf{m}) \rho(\mathbf{r})}^{\gamma(\mathbf{q})} \\
& \left.+\sum_{r_{f}>0}\left(C_{\mu(\mathbf{m}) \rho(\mathbf{r})}^{\gamma(\mathbf{q})} C_{\nu(\mathbf{n}) \tau(\mathbf{t})}^{\rho(\mathbf{r})}-C_{\rho(\mathbf{r}) \tau(\mathbf{t})}^{\gamma(\mathbf{q})} C_{\mu(\mathbf{m}) \nu(\mathbf{n})}^{\rho(\mathbf{r})}\right)\right]\left|l^{\mu}(\mathbf{m}) l^{\nu}(\mathbf{n})\right\rangle+\ldots \\
& \tilde{X}_{l^{\tau}(\mathbf{t})_{\left(t_{f} \leq 0\right)}^{R}}^{R}\left|s^{\gamma}(\mathbf{q})\right\rangle=C_{\tau(\mathbf{t}) \mu(\mathbf{m})}^{\tilde{\gamma}(\mathbf{q})}\left|l^{\mu}(\mathbf{m})\right\rangle+C_{\tau(\mathbf{t}) \tilde{\nu}(\mathbf{n})}^{\tilde{\gamma}(\mathbf{q})}\left|s^{\nu}(\mathbf{n})\right\rangle \\
& +\quad C_{\tau(\mathbf{t}) \nu(\mathbf{n})}^{\varphi}\left|l^{\nu}(\mathbf{n}) s^{\gamma}(\mathbf{q})\right\rangle+C_{\tau(\mathbf{t}) \tilde{\nu}(\mathbf{n})}^{\varphi}\left|s^{\nu}(\mathbf{n}) s^{\gamma}(\mathbf{q})\right\rangle \\
& -\left[\sum_{r_{f} \leq 0}\left(\frac{3}{8} C_{\tau(\mathbf{t}) \mu(\mathbf{m})}^{\rho(\mathbf{r})} C_{\nu(\mathbf{n}) \rho(\mathbf{r})}^{\tilde{\gamma}(\mathbf{q})}+\frac{3}{4} C_{\tau(\mathbf{t}) \mu(\mathbf{m})}^{\tilde{\rho}(\mathbf{r})} C_{\nu(\mathbf{n}) \tilde{\rho}(\mathbf{r})}^{\tilde{\gamma}(\mathbf{q})}\right)\right. \\
& +\sum_{r_{f}>0}\left(\frac{1}{8} C_{\mu(\mathbf{m}) \rho(\mathbf{r})}^{\tilde{\gamma}(\mathbf{q})} C_{\nu(\mathbf{n}) \tau(\mathbf{t})}^{\rho(\mathbf{r})}+\frac{1}{2} C_{\mu(\mathbf{m}) \tilde{\rho}(\mathbf{r})}^{\tilde{\gamma}(\mathbf{q})} C_{\nu(\mathbf{n}) \tau(\mathbf{t})}^{\tilde{\rho}(\mathbf{r})}\right. \\
& \left.\left.-\quad \frac{1}{8} C_{\rho(\mathbf{r}) \tau(\mathbf{t})}^{\tilde{\gamma}(\mathbf{(})} C_{\mu(\mathbf{m}) \nu(\mathbf{n})}^{\rho(\mathbf{r})}\right)\right]\left|l^{\mu}(\mathbf{m}) l^{\nu}(\mathbf{n})\right\rangle+C_{\tau(\mathbf{t}) \mathbf{n}}^{\varphi}\left|a(\mathbf{n}) s^{\gamma}(\mathbf{q})\right\rangle \\
& +\sum_{r_{f} \leq 0}\left(C_{\tilde{\nu}(\mathbf{n}) \tau(\mathbf{t})}^{\tilde{\rho}(\mathbf{r})} C_{\mu(\mathbf{m}) \tilde{\rho}(\mathbf{r})}^{\tilde{\gamma}(\mathbf{q})}\left|l^{\mu}(\mathbf{m}) s^{\nu}(\mathbf{n})\right\rangle+\frac{1}{2} C_{\mathbf{n} \tau(\mathbf{t})}^{\mathbf{r}} C_{\mathbf{m} \mathbf{r}}^{\tilde{\gamma}(\mathbf{q})}|a(\mathbf{m}) a(\mathbf{n})\rangle\right)+\ldots \\
& \tilde{X}_{l^{\tau}(\mathbf{t})_{\left(t_{f} \leq 0\right)}}^{R}|a(\mathbf{q})\rangle=C_{\tau(\mathbf{t}) \mathbf{n}}^{\mathbf{q}}|a(\mathbf{n})\rangle+C_{\tau(\mathbf{t}) \nu(\mathbf{n})}^{\varphi}\left|l^{\nu}(\mathbf{n}) a(\mathbf{q})\right\rangle+\frac{1}{2} C_{\tau(\mathbf{t}) \tilde{\nu}(\mathbf{n})}^{\varphi}\left|s^{\nu}(\mathbf{n}) a(\mathbf{q})\right\rangle \\
& +C_{\tau(\mathbf{t}) \mathbf{n}}^{\varphi}|a(\mathbf{n}) a(\mathbf{q})\rangle-\sum_{r_{f} \leq 0} C_{\tau(\mathbf{t}) \mathbf{n}}^{\mathbf{r}} C_{\mu(\mathbf{m}) \mathbf{r}}^{\mathbf{q}}\left|a(\mathbf{n}) l^{\mu}(\mathbf{m})\right\rangle+\ldots \\
& \tilde{X}_{a(\mathbf{t})_{\left(t_{f}>0\right)}^{R}}^{R}|0\rangle=0
\end{aligned}
$$




$$
\begin{aligned}
& \tilde{X}_{a(\mathbf{t})_{\left(t_{f}>0\right)}}^{R}|a(\mathbf{q})\rangle=\delta(\mathbf{q}-\mathbf{t})|0\rangle \\
& \tilde{X}_{a(\mathbf{t})_{\left(t_{f}>0\right)}}^{R}\left|l^{\gamma}(\mathbf{q})\right\rangle=0 \\
& \tilde{X}_{a(\mathbf{t})_{\left(t_{f}>0\right)}^{R}}^{R}\left|s^{\gamma}(\mathbf{q})\right\rangle=0 \\
& \tilde{X}_{s^{\tau}(\mathbf{t})_{\left(t_{f}>0\right)}^{R}}^{R}|0\rangle=0 \\
& \tilde{X}_{s^{\tau}(\mathbf{t})_{\left(t_{f}>0\right)}}^{R}|a(\mathbf{q})\rangle=0 \\
& \tilde{X}_{s^{\tau}(\mathbf{t})_{\left(t_{f}>0\right)}^{R}}^{R}\left|l^{\gamma}(\mathbf{q})\right\rangle=0 \\
& \tilde{X}_{s^{\tau}(\mathbf{t})_{\left(t_{f}>0\right)}^{R}}\left|s^{\gamma}(\mathbf{q})\right\rangle=\delta_{\tau}^{\gamma} \delta(\mathbf{q}-\mathbf{t})|0\rangle \\
& \tilde{X}_{l^{\tau}(\mathbf{t})_{\left(t_{f}>0\right)}^{R}}^{R}|0\rangle=0 \\
& \tilde{X}_{l^{\tau}(\mathbf{t})_{\left(t_{f}>0\right)}^{R}}^{R}|a(\mathbf{q})\rangle=0 \\
& \tilde{X}_{l^{\tau}(\mathbf{t})_{\left(t_{f}>0\right)}^{R}}\left|l^{\gamma}(\mathbf{q})\right\rangle=\delta_{\tau}^{\gamma} \delta(\mathbf{q}-\mathbf{t})|0\rangle \\
& \tilde{X}_{l^{\tau}(\mathbf{t})_{\left(t_{f}>0\right)}^{R}}^{R}\left|s^{\gamma}(\mathbf{q})\right\rangle=0 \text {, }
\end{aligned}
$$

where $|0\rangle$ behaves as a vacuum state and is unique by construction. The action on higher-order functions is limited by the initial perturbative development in the group law. Apart from that, its computation is completely straightforward, though highly tedious.

As we said in the Introduction, the Hilbert product $\langle\ldots \mid \ldots\rangle$ is introduced by imposing $\langle 0 \mid 0\rangle=1$ and specifying the adjoint for each operator. In order to do that, we set the notation

$$
\hat{L}_{\mu}(\mathbf{m})=\tilde{X}_{l^{\mu}(\mathbf{m})}^{R}, \hat{A}(\mathbf{m})=\tilde{X}_{a(\mathbf{m})}^{R}, \hat{S}^{\rho}(\mathbf{m})=\tilde{X}_{s_{\rho}(\mathbf{m})}^{R},
$$

and stablish the corresponding adjoint operators

$$
\hat{L}_{\mu}^{\dagger}(\mathbf{m})=\hat{L}_{\mu}(-\mathbf{m}), \hat{A}^{\dagger}(\mathbf{m})=\hat{A}(-\mathbf{m}),\left(\hat{S}^{\rho}\right)^{\dagger}(\mathbf{m})=\hat{S}^{\rho}(-\mathbf{m}) .
$$

\section{Physical interpretation}

As we mentioned in the Introduction, in the higher-dimensional case we no longer have the connection with Physics provided by the Polyakov action. This is the reason why we have not developed the analysis of the semi-classical situation nor constructed a Lagrangian, limiting ourselves to the presentation of the Noether invariants defining the symplectic structure. Therefore, the link between the abstract group parameters and the metric ingredients, needed to make contact with a gravitational theory, is less obvious. In fact, the present model is more properly interpreted as a kind of topological theory, though with local degrees of freedom, where a metric notion must be recovered by imposing a constraint (in the spirit of General relativity from BF theory [20, 21]) or by adding an extra structure.

We present here different situations framed in a gravitational setting where the mathematical construction we have developed plays a significant role. 


\section{Space-time with boundary conditions}

Perhaps the most conservative application of the mathematical formalism developed in the previous section takes place in the context of standard General Relativity when boundary conditions play a significant role. The study of the solution space of the (classical) theory in these scenarios shows that, due to the presence of these conditions, some of the diffeomorphisms are not generated by the constraints of the theory [22] and therefore the former are not gauge transformations. As a consequence, they actually move the solution space and constitute a dynamical symmetry. In fact, in some models like asymptotic Anti de Sitter solutions to $2+1$ gravity [23, 24], which can be treated as a Chern-Simmons theory, or some treatments of black hole entropy [25] these diffeomorphism parametrize the phase space of the theory providing the physical degrees of freedom. This identification of the solution space is a crucial first step for the quantization of the theory.

Abstract models for diffeomorphism dynamics like the one presented above could prove to be useful in situations where the presence of more general boundary conditions confers a dynamical content to other subgroups of the diffeomorphism symmetry. One cannot avoid the impression that the limits in the application of these kind of techniques to more general scenarios is more related to our poor technical skills beyond the Virasoro symmetry than to actual conceptual reasons.

\section{Hilbert Space of a Gravitational Theory}

In the construction of a dynamical system out of a symmetry at the (semi-)classical level, there is a fundamental difference between the GAQ and, for instance, a more standard coadjoint orbits method. In fact, the former provides not only a symplectic structure on which dynamics can be described, but also determines the Hamiltonian function(s) which specifies the evolution of the system. In this sense, our topological model is endowed with an intrinsic dynamics dictated by the vector fields in the characteristic module $\mathcal{G}_{\Theta}$.

Nevertheless, we need not the full structure of GAQ to formulate well-defined models. We can resort to the GAQ as a powerful method to obtain, out of a fundamental symmetry, a welldefined phase space on which dynamics are introduced by suplementing some extra information.

In this spirit, we are going to look at the quantum representation space of the previous section as the Hilbert space for a genuine quantum gravitational theory. In this sense, our mathematical construction supplies the kinematics of such a Gravity model. The relevance of this Hilbert space for a gravitational theory relies on the fact that the operators $L_{\mu}^{\dagger}(\mathbf{m})$ and $L_{\mu}(\mathbf{m})$ essentially constitute a quantum realization of the vector fields with support on a manifold $M$. Taking advantage of the fact that a classical metric field is a symmetric $(0,2)$ tensor field characterized by its action on such vector fields, we are introducing a quantum metric operator $\hat{g}$ by defining its action on some appropiate states ("quantized vector fields" on a manifold). An appropriate set of vector fields is the one defining a tetrad basis $\left\{e_{a}\right\}$. Considering the components of these tetrads in a coordinate basis and formally expanding them in plane-waves, we find,

$$
e_{a}(x)=e_{a}^{\mu}(x) \partial_{\mu}=\sum_{\mathbf{m}} e_{a}^{\mu}(\mathbf{m}) e^{i \mathbf{m}_{\rho} x^{\rho}} \partial_{\mu}=\sum_{\mathbf{m}} e_{a}^{\mu}(\mathbf{m}) L_{\mu}(\mathbf{m})
$$

where we have made used of the notation introduced in subsection 2.2. Then, we promote the classical field $L_{\mu}(\mathbf{m})$ to a quantum operator by using the representation constructed in section 
3,

$$
e_{a} \mapsto e_{a}^{\mu}(\mathbf{0}) \hat{L}_{\mu}^{\dagger}(\mathbf{0})+\sum_{m_{f}>0}\left(e_{a}^{\mu}(-\mathbf{m}) \hat{L}_{\mu}^{\dagger}(\mathbf{m})+e_{a}^{\mu}(\mathbf{m}) \hat{L}_{\mu}(\mathbf{m})\right)
$$

Associating with each of this operators a canonical state, $\left|e_{a}\right\rangle \equiv \hat{e}_{a}|0\rangle$, we construct a metric operator $\hat{g}$ by imposing the quantum tetrad condition,

$$
\left\langle e_{a}|\hat{g}| e_{b}\right\rangle=\eta_{a b}
$$

A minimal manner (in the sense that more general approaches could be followed) of implementing this condition consists in restricting the domain of the operator $\hat{g}$ to one-particle states $L_{\mu}^{\dagger}(\mathbf{m})|0\rangle$. Even more, we demand it to be hermitian $\left(\hat{g}^{\dagger}=\hat{g}\right)$ and diagonal on the $\mathbf{m}$ label of these states: $\hat{g} L_{\mu}^{\dagger}(\mathbf{m})|0\rangle=g_{\mu}^{\nu}(\mathbf{m}) L_{\nu}^{\dagger}(\mathbf{m})|0\rangle$. In order to make a comparison with the classical theory, it is better to introduce an object covariant in the two space-time indices, $g_{\mu \nu}(\mathbf{m}) \equiv\left\langle 0\left|L_{\mu}(\mathbf{m}) \hat{g} L_{\nu}(\mathbf{m})\right| 0\right\rangle$, whose explicit form is

$$
g_{\mu \nu}(\mathbf{m})=\frac{-C_{\rho}}{2}\left(g _ { \mu } ^ { \sigma } \left(\mathbf{m}\left(m_{\nu} \delta_{\sigma}^{\rho}+m_{\sigma} \delta_{\nu}^{\rho}\right)+g_{\nu}^{\sigma}\left(\mathbf{m}\left(m_{\mu} \delta_{\sigma}^{\rho}+m_{\sigma} \delta_{\mu}^{\rho}\right)\right),\right.\right.
$$

where we have considered for simplicity that the only non-vanishing central parameter in (9) is $C_{\rho}$. With these elements, condition (26) simply expresses:

$$
\eta_{a b}=\sum_{\mathbf{m}} g_{\mu \nu}(\mathbf{m}) e_{a}^{\mu}(\mathbf{m}) e_{b}^{\nu *}(\mathbf{m})
$$

which is the analogue in this simplified example to the standard space-time variables expression and relates the quantum action of $\hat{g}$ with a metric notion.

The connection with the classical theory can be achieved by reversing the step $L_{\mu}(\mathbf{m}) \mapsto$ $\hat{L}_{\mu}(\mathbf{m})$, and taking the parameters $g_{\mu \nu}(\mathbf{m})$ as the Fourier components of the classical metric field:

$$
g_{\mu \nu}(x)=\sum_{\mathbf{m}} e^{i m_{\rho} x^{\rho}} g_{\mu \nu}(\mathbf{m})
$$

As we said before, the previous discussion describes the kinematics of the metric degrees of freedom, but offers no clue on the way in which the specific $g_{\nu}^{\mu}(\mathbf{m})\left(\right.$ or $\left.e_{a}^{\mu}(\mathbf{m})\right)$ should be chosen. This is a dynamical information that will be added in this approach by imposing a constraint, external to the group, on the $g_{\mu \nu}(\mathbf{m})$ parameters. The simplest one we can imagine, having a straightforward link to classical physics, is the Fourier transform of Einstein equations in vacuum, $R_{\mu \nu}=0$. We find,

$$
\begin{array}{r}
0=m_{\lambda} \sum_{\mathbf{m}} g^{\lambda \sigma}(\mathbf{m}-\mathbf{n})\left(n_{\nu} g_{\mu \sigma}(\mathbf{n})+n_{\mu} g_{\nu \sigma}(\mathbf{n})-n_{\sigma} g_{\nu \mu}(\mathbf{n})\right)- \\
-n_{\nu} \sum_{\mathbf{n}} g^{\lambda \sigma}(\mathbf{m}-\mathbf{n})\left(n_{\lambda} g_{\mu \sigma}(\mathbf{n})+n_{\mu} g_{\lambda \sigma}(\mathbf{n})-n_{\sigma} g_{\nu \mu}(\mathbf{n})\right)+ \\
+\sum_{\mathbf{n}, \mathbf{p}, \mathbf{q}} g^{\eta \sigma}(\mathbf{m}-\mathbf{n}-\mathbf{p})\left(p_{\nu} g_{\mu \sigma}(\mathbf{p})+p_{\mu} g_{\nu \sigma}(\mathbf{p})-p_{\sigma} g_{\nu \mu}(\mathbf{p})\right) \\
\quad g^{\lambda \sigma^{\prime}}(\mathbf{n}-\mathbf{q})\left(q_{\lambda} g_{\eta \sigma^{\prime}}(\mathbf{q})+q_{\eta} g_{\lambda \sigma^{\prime}}(\mathbf{q})-q_{\sigma^{\prime}} g_{\lambda \eta}(\mathbf{q})\right)- \\
-\sum_{\mathbf{n}, \mathbf{p}, \mathbf{q}} g^{\eta \sigma}(\mathbf{m}-\mathbf{n}-\mathbf{p})\left(p_{\lambda} g_{\mu \sigma}(\mathbf{p})+p_{\mu} g_{\lambda \sigma}(\mathbf{p})-p_{\sigma} g_{\lambda \mu}(\mathbf{p})\right) \\
g^{\lambda \sigma^{\prime}}(\mathbf{n}-\mathbf{q})\left(q_{\nu} g_{\eta \sigma^{\prime}}(\mathbf{q})+q_{\eta} g_{\nu \sigma^{\prime}}(\mathbf{q})-q_{\sigma^{\prime}} g_{\nu \eta}(\mathbf{q})\right)
\end{array}
$$


A model where (a sector of) the quantum phase space is constructed out of the diffeomorphism modes, corresponds to a theory where (part of) the diffeomorphisms are not gauge transformations at the quantum level. We can then interpret the states in the Hilbert space as physical distortions of a reference space-time corresponding to the maximum weight vector in the representation. Therefore, $|0\rangle$ would correspond to a locally flat space-time, where locally refers here to an entire neighbourhood, not only to a point. One-particle states, which display a direct link with the quantized vector fields, would correspond to local excitations of the metric field in a given space-time direction, creating a distortion in distances measured by the action of the quantum operator $\hat{g}$. Even tough the restricted definition of $\hat{g}$ to only the linear subspace of oneparticle states is enough to show quantum features such as the probability transition between different metric excitation states, one can think of endowing multi-particle states also with the metric content. A simple way of extending the domain of $\hat{g}$ consists in defining a proyector $P$ $\left(P^{2}=P\right)$ from the total representation Hilbert space to that of mono-particular states. Then, we define $\hat{g}|\Psi\rangle \equiv \hat{g} P|\Psi\rangle$, so that an arbitrary state acquires a metric excitation interpretation.

\section{Gauge Gravity Theories}

In the GAQ, the group law of the starting symmetry comprises both the connection fields parameters and the ones corresponding to the local or gauge group itself. However, and similarly to the shown electromagnetic case, a subtle non-trivial mixing between these two sets of parameters occurs which implies a restructuration of the gauge and physical degrees of freedom. As a result of this process, the original elements of the gauge group do gain a dynamical content. When approaching gravity by using local diffeomorphisms as the symmetry associated with local translations, the $l^{\mu}(\mathbf{m})$ parameters themselves acquire a metric meaning. Of course, the complete theory needs the addition of the other part of the group, something that could be faced in principle in different ways (directly metric structures, tetrad fields, Ashtekar connection variables...). But independently on the actual way in which this missing structure is introduced, combinations of $m_{\mu} \tilde{X}_{l^{\nu}(\mathbf{m})}^{R}$ will enter the theory as part of the physical gravitational degrees of freedom. Apart from that, the presence of new fields like $S^{\rho}(\mathbf{m})$ in the extended diffeomorphism group, opens the possibility of constructing the matter side of the Einstein-like equations out of the symmetry structure. In the spirit of a gauge approach to Gravity, the construction in section 3 must be understood as an indermediate step in the development of a yet-to-be-completed theory. Regarding the breakdown of diffeomorphism invariance, the presence of central terms in the commutator of the diffeomorphism generators turn then into a set of second-class contraints. Not all the spurious degrees of freedom can be eliminated, thus appearing new physical modes. This is analogous to the emergence of Proca field in [5] or to the mechanism for cohomological mass generation of gauge bosons in [6]. Finally, the secondary goal of advancing in the understanding of the interaction mixing is accomplished by making explicit the non-trivial coupling of the diffeomorphisms and the local $U(1)$ symmetry. In a general sense beyond the specific GAQ, the insight into diffeomorphism representations provided in section 3, will help the understanding of the imposition of diffeomorphism constraints in diffeomorphism invariant theories where a gauge is not fixed prior to quantization.

\section{Phenomenological Theories}

The last physical interpretation promotes our construction to an effective theory valid at lowintermediate energies. An underlying more fundamental theory is assumed to exist at higher 
energies, with the only constraint that diffeomorphism invariance, as a gauge symmetry, must play a fundamental role in its formulation. Lacking such a description, though candidates do exist, a phenomenological one is proposed leaning on two basic assumptions. On the one hand, the specific space-time dynamics are decoupled and described by the (semi-)classical theory (General Relativity or, more generally, Quantum Field Theory on curved spaces) and, on the other hand, the diffeomorphism invariance is anomalously realized in the quantum process which describes the implementation of the matter fields. This anomalous realization gives rise to effective deformations of the fundamental diffeomorphism algebra $\left(S^{\mu}(\mathbf{m})\right.$ field) and confers with a dynamical content to some of the, in principle spurious, gauge diffeomorphism degrees of freedom (the implementation of the diffeomorphism gauge invariance breakdown is explicit here). In this sense, the occurrence of an anomaly reveals the presence of more fundamental underlying physics at higher energies whose degrees of freedom are encoded at these intermediate energies in the emergent effective degrees of freedom [26]. The particular model in this paper can be seen as a generalization to higher dimensions of the example presented in [27]. This reference provides a more detailed description of the physics involved in this approach and suggests a mechanism for particle mass generation. Identifying $\hat{L}_{\mu}(\mathbf{0})$ with the Hamiltonian of the complete physical system, in a given coordinate system, and looking at the explicit form of its associated Noether invariant in terms of those ones corresponding to the physical degrees of freedom (see (16)), we find the general form

$$
\hat{H}_{\text {eff }}=\hat{H}_{\text {free }}\left(\hat{\Phi}^{\dagger}, \hat{\Phi}\right)+\hat{H}_{\text {mixing }}\left(\hat{\Phi}^{\dagger}, \hat{\Phi},\left(\hat{L}_{\mu}\right)^{\dagger}, \hat{L}_{\mu},(\hat{F})^{\dagger}, \hat{F}\right)
$$

where $\hat{\Phi}$ denote the non-gravitational fundamental degrees of freedom (here $\hat{A}$, which can be seen as a scalar field), $\hat{L}_{\mu}$ are the dynamical diffeomorphisms and $\hat{F}$ are the extra anomalous effective degrees of freedom ( $\hat{S}^{\mu}$ in our case). As we can see, the presence of such an anomaly entails corrections to the spectrum of the fundamental non-gravitational degrees of freedom, opening the above-mentioned possibility of relating the emergence of mass terms to them. Finally, we must point out that in contrast with the first physical interpretation, this phenomenological approach takes full advantage of the structures obtained with the GAQ. In fact, not only a representation space is constructed but also the dynamics is determined by the choice of the Hamiltonian among the generators inside $\mathcal{G}_{\Theta}$.

\section{Conclusions}

The main result of the present work is the construction of a maximum-weight representation corresponding to the infinite-dimensional Lie algebra closed by the local diffeomorphisms $L_{\mu}(\mathbf{m})$ defined on a manifold of dimension superior to one, acting in a semi-direct way on scalar modes $A(\mathbf{m})$ and finally non-centrally extended by the generator $S^{\rho}(\mathbf{m})$. Although this kind of representations are already studied in [28], the singularity of ours is related to its explicit (non-formal) character and to the central role played by the concept of pseudo-cohomology. On the other hand, it fulfills the generalization of the mathematical structure in [1].

The physical relevance of this construction in the setting of (quantum) gravitational dynamics is exemplified by the discussion of several different approaches to the general problem. They all share the feature of confering a priviledged role to the notion of symmetry in the construction of the physical system. Our main conclusion is that the diffeomorphism group by its own cannot account for the complete gravitational dynamics (the basic aim in [1]), since 
it basicly defines a kind of topological theory that needs to be suplemented with additional structure (either including it inside a bigger group, or by imposing a constraint, etcetera) in order to make contact with metric notions. However, this algebra proves to play a key role in the formulation of some non-trivial physical models for quantum gravity, where the issues of quantum diffeomorphism invariance breakdown and the mixing with internal symmetries are raised. They suggest, in fact, some potential experimental consequences such as the presence of extra fields corresponding to non-central extensions $\left(S^{\rho}\right)$ or the corrections to non-gravitational fields propagators (phenomelogical model).

\section{Acknowledgements}

One of us, J.L.J. would like to thank the Mathematical Department of the Queen Mary and Westfield College (University of London), and especifically to Prof. S. Majid, for the hospitality during the development of part of the work.

\section{References}

[1] V. Aldaya, J.L. Jaramillo, Class. Quant. Grav. 17, 1649 (2000).

V. Aldaya, J.L. Jaramillo, Class. Quant. Grav. 17, 4877 (2000).

[2] A.M. Polyakov, Mod. Phys. Lett. A11, 893 (1987).

[3] V. Aldaya, J. Navarro-Salas, A. Ramírez, Commun. Math. Phys., 121, 541 (1989).

[4] V. Aldaya, J.L. Jaramillo and J. Guerrero, to appear in J. Phys A, hep-th/0105278.

V. Aldaya, J.L. Jaramillo and J. Guerrero, submitted.

[5] V. Aldaya, M. Calixto, M. Navarro, Int.J.Mod.Phys. A12, 3609 (1997).

[6] M. Calixto, V. Aldaya, J. Phys. A 32, 7287 (1999).

[7] T.W.B. Kibble, J. Math. Phys. 2, 212 (1961).

[8] E. Álvarez, Rev. Mod. Phys. 61, 561 (1989).

[9] N.M.J. Woodhouse, Geometric Quantization, Oxford University Press, (1991).

[10] D.B. Fuks, Cohomology of infinite dimensional algebras, Consultants Bureau (Russian ed. (1984)), 1986

[11] V. Aldaya, J. Guerrero, Rep. Math. Phys. 47, 213 (2001).

[12] T.A. Larsson, math-ph/0002016.

[13] A. Dzhumadil'daev, Z. Phys. C 72, 509 (1996).

[14] R. Utiyama, Phys. Rev. 101, 1597 (1956).

[15] J.P. Serre, Lie algebras and Lie groups, New Yor, Benjamin (1965).

[16] V. Aldaya, J. Navarro-Salas, Commun. Math. Phys. 113, 375 (1987). 
[17] E.J. Saletan, J. Math. Phys. 2, 1 (1961).

[18] V. Aldaya, J. A. de Azcárraga, Int. J. Theo. Phys. 24, 141 (1985).

[19] V. Aldaya, J. Navarro-Salas, Commun. Math. Phys. 113375 (1987).

[20] J. Plebanski, J. Math. Phys. 18, 2511 (1977).

[21] R. Capovilla, J. Dell, T. Jacobson, Class. Quant. Grav 8, 59 (1991).

[22] T. Regge, C. Teitelboim, Ann. Phys. (N.Y.) 88, 286 (1974).

[23] J.D. Brown, M. Henneaux, Commun. Math. Phys. 104, 207 (1986).

[24] M. Bañados, Charla invitada en Second Meeting "Trends in Theoretical Physics", Buenos Aires (1998); hep-th9901148.

[25] S. Carlip, Nucl. Phys. Proc. Suppl. 57, 8 (1997).

[26] J. Preskill, Ann. Phys.(N.Y.) 210, 323 (1991).

[27] J.L. Jaramillo, V. Aldaya, Mod. Phys. Lett. A17, 809 (2002).

[28] T.A. Larsson, Commun. Math. Phys. 201 (1999), 461. 